\title{
Topological defects around a spherical nanoparticle in nematic liquid crystal: Coarse-grained molecular dynamics simulations
}

Cite as: J. Chem. Phys. 141, 114903 (2014); https://doi.org/10.1063/1.4894438

Submitted: 26 May 2014 • Accepted: 12 August 2014 • Published Online: 15 September 2014

Jaroslav M. Ilnytskyi, Andrij Trokhymchuk and Martin Schoen

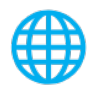

\section{ARTICLES YOU MAY BE INTERESTED IN}

Defects in liquid crystals

Physics Today 35, 48 (1982); https://doi.org/10.1063/1.2915094

Effects of flow on topological defects in a nematic liquid crystal near a colloid

The Journal of Chemical Physics 140, 054905 (2014); https://doi.org/10.1063/1.4862953

Nanoparticles in nematic liquid crystals: Interactions with nanochannels

The Journal of Chemical Physics 127, 124702 (2007); https://doi.org/10.1063/1.2770724

The Journal

SPECIAL TOPIC: Low-Dimensional

of Chemical Physics Materials for Quantum Information Science 


\title{
Topological defects around a spherical nanoparticle in nematic liquid crystal: Coarse-grained molecular dynamics simulations
}

\author{
Jaroslav M. Inytskyi, ${ }^{1, a)}$ Andrij Trokhymchuk, ${ }^{1, b)}$ and Martin Schoen ${ }^{2, c)}$ \\ ${ }^{1}$ Institute for Condensed Matter Physics of the National Academy of Sciences of Ukraine, 1, Svientsitskii Str., \\ 79011 Lviv, Ukraine \\ ${ }^{2}$ Stranski-Laboratorium für Physikalische und Theoretische Chemie, Fakultät für Mathematik und \\ Naturwissenschaften, Technische Universität Berlin, Straße des 17. Juni 135, 10623 Berlin, Germany and \\ Department of Chemical and Biomolecular Engineering, Engineering Building I, Box 7905, North Carolina \\ State University, 911 Partners Way, Raleigh, North Carolina 27695, USA
}

(Received 26 May 2014; accepted 12 August 2014; published online 15 September 2014)

\begin{abstract}
We consider the applicability of coarse-grained molecular dynamics for the simulation of defects in a nematic liquid crystal around a colloidal particle. Two types of colloids are considered, a soft colloid resembling a liquid crystal dendrimer or a similar macromolecule. In addition, a decorated colloid is used which could represent a gold nanoparticle with mesogen-modified surface. For both models we consider homeotropic and tangential anchoring. Precise control of the easy axis on the colloid's surface enables us to focus on specific planar arrangements in the case of a decorated colloid. The nematic phase is modelled explicitly via soft spherocylinders interacting through a potential, suggested by Lintuvuori and Wilson [J. Chem. Phys. 128, 044906 (2008)]. Properties of the nematic phase are studied by computing the Frank elastic constants. In addition, estimates for the nematic-isotropic transition and the coherence length allow us to establish a relation between energy and length scales with respect to experimental systems. Both models exhibit similar defect topologies, namely, that of a Saturn ring and a boojum-type of defect for homeotropic and tangential surface anchoring, respectively. In the decorated colloid model we tune the anchoring strength through the density of the mesogenic shell on the surface. We also found the biaxial boojum defect for the special case of longitudinal planar anchoring. The study demonstrates the potential of coarse-grained simulation methods for studying defects in liquid crystals. () 2014 AIP Publishing LLC. [http://dx.doi.org/10.1063/1.4894438]
\end{abstract}

\section{INTRODUCTION}

Colloidal particles dispersed in a liquid crystalline (LC) environment are a fascinating class of soft matter ${ }^{1}$ composite materials with rich physics and inexhaustible potential for technological innovation. ${ }^{2-4}$ Nowadays so-called liquid crystal colloids (LCCs) form the basis for advanced functional and structural materials, engineering, and household devices and products, and play a crucial role in various LC based industrial applications. By comparing LCC dispersions with situations in which the LC host phase has been replaced by an ordinary liquid, one realises that it is the LC medium that makes the LCC materials so unique. Therefore, understanding properties of the LC host in the presence of the colloidal particles represents a fundamental problem for LC science and technology.

Although the LC state of matter has been observed for the first time more than one and a half centuries ago by Planer (reprinted in Ref. 5), the interest in a deeper understanding of LCs and their properties was stimulated only fairly recently by a growing number of technological applications. From a modern point of view, the unique properties of the LC medium are determined by the anisotropy of

\footnotetext{
a) Electronic mail: iln@icmp.lviv.ua

b) Electronic mail: adt@icmp.lviv.ua

c)Electronic mail: martin.schoen@tu-berlin.de
}

long-range and/or short-range intermolecular interactions. For certain temperatures (pressures) these anisotropic interactions result in long-range orientational ordering of the long axes of the LC molecules (mesogens) along a preferred direction, the so-called director. At the interface between a colloidal particle and the LC host local perturbations of the director occur, resulting in topological defects in the vicinity of the colloid. If more than a single colloid is present these defects give rise to effective interactions between them. Such LC-mediated interactions have no analogue if the colloids are dispersed in an isotropic medium. Hence, the properties of LCC materials such as colloidal self-assembly are the immediate consequence of the effective interactions.

The energy scale of these LC-mediated colloid-colloid interactions is found to depend on both the size of colloid particles and on the strength of the orientational anchoring on their surface. Typical values of energy for the case of micronsize colloids is several hundreds of $k_{\mathrm{B}} T^{6-8}$ ( $k_{\mathrm{B}}$ Boltzmann's constant, $T$ temperature). However, as shown in Ref. 9, similar values can be achieved for much smaller colloid sizes of $125-500 \mathrm{~nm}$ with suitable surface functionalization. It is evident that the principal factor that defines the strength of LCmediated colloid-colloid interaction is the degree of distortion of the director near the colloid's surface.

The distortion of the director field near an isolated spherical particle has been addressed by many researchers 
during the last $10-15$ years. ${ }^{10-13}$ To be more specific, direct experimental measurements were performed by Poulin et $a l .{ }^{14}$ Continuum theory, where one minimizes the FrankOseen ${ }^{10,15,16}$ or the Landau-de Gennes free energy, ${ }^{17}$ was developed, including the use of various ansatz functions. ${ }^{16,18}$ Monte Carlo ${ }^{19-21}$ as well as molecular dynamics ${ }^{22-24}$ simulations have been conducted using a range of models. For colloids with homeotropic orientation of mesogens at the surface, two types of topological defects may arise. ${ }^{10}$ One of these is the so-called hyperbolic hedgehog defect. Typically, it is observed in micron-sized colloids, and the asymptotic behavior of the director field in this case has dipolar symmetry. The other defect is the so-called Saturn ring defect where the disclination line encircles the colloid. The director field in this case has quadrupolar symmetry. This defect (and its limiting case of a surface ring observed in the case of weak anchoring), is stable for nanometer-sized colloids. For the case of tangential anchoring, the boojum defect topology is reported. ${ }^{21,25,26}$

Computer simulations with explicit modelling of the LC host have a benefit of being able to address the positions and orientations of each mesogen. This results in the ability to reproduce fine details of the structure of the director field near the colloid's surface. However, accessible length and time scales of the simulated system are restricted and, as a result, a study of micrometer-sized colloid case is nearly impossible. Several molecular models for the LC medium have been considered to study the LCCs with molecular simulations so far. Perhaps, the most popular one is that originally proposed by Gay and Berne (GB), ${ }^{27}$ which is a modification of the standard 12-6 Lennard-Jones potential for prolate and oblate ellipsoidal particles. The GB model is effective for modeling mesogenic systems, comprising both the short-range (shape) and long-range anisotropic interactions. The WeeksChandler-Andersen simplification of the GB model, which neglects attractive forces between ellipsoidal molecules, was also used to simulate LC molecules. ${ }^{23}$ An even simpler model has been suggested by Hess and $\mathrm{Su}^{28}$ and employed in Ref. 21. It consists of the Lennard-Jones potential in which only the attractive contribution has been modified to represent the orientation-dependent interaction between slightly elongated mesogens.

To extend accessible length and time scales of the simulation, one may turn one's attention to coarse-grained models. Relatively recently, the soft-core spherocylinder model was introduced for the simulations of LC mesophases by Lintuvuori and Wilson. ${ }^{29}$ It has already been used in a number of studies of LC oligomers and macromolecules. ${ }^{30-34}$ In principle, the coarse-grained nature of this model should allow one to explore larger system sizes, required for the description of certain defect topologies and has a potential to speed up the equilibration of the system. We perform a thorough analysis of the applicability of this soft-core spherocylinder model for the simulation of defects around an isolated spherical colloid particle. We begin by studying properties of the nematic LC host phase. These include the isotropic-nematic transition temperature, the Frank elastic constants, and the coherence length of the mesogen-mesogen pair interaction. This allows us to establish a link between our model system and an experimental LC. The models for a coarse-grained representation of the colloid are discussed, emphasizing the ability to vary the type of anchoring and its strength. The latter is related to the radial coherence length for the colloid-mesogen interaction.

The reminder of the paper is organized as follows. In Sec. II we discuss concepts for modelling soft and decorated colloids. In Sec. III we focus on properties of the nematic phase formed by soft-core spherocylinders. Section IV contains results for defects around a soft colloid with homeotropic and planar anchoring, whereas a similar study for the case of a decorated colloid is covered by Sec. V. Finally, we draw conclusions of this study in Sec. VI.

\section{CONCEPTS OF MESOSCOPIC MODELLING}

In this study we concentrate on the distortion of the director field near the surface of a colloid. Focusing on the mesoscale level, two assumptions are made from the outset:

(i) the colloid is spherical and keeps its shape by means of interactions between its constituents;

(ii) the colloid's surface anchors adjacent mesogens of the LC host phase such that these mesogens assume a specific orientation with respect to the colloid's local surface normal.

Based on these two premises one may envision a broad range of experimental realisations some of which are illustrated in Fig. 1. For example, case (a) depicts a solid (e.g., gold $^{35,36}$ ) nanoparticle with surfaces modified by grafted mesogens. The sphericity of such a colloid is ensured by the impenetrable solid core, whereas the surface anchoring strength is controlled by the grafting density and the length of a connecting spacer. We refer to this situation as "decorated colloid." Two other cases in Fig. 1 represent softer objects with no hard core, which will be referred to as "soft colloids." In particular, case (b) shows a carbosilane dendrimer with its surface formed by a shell of mesogens. We consider the high density limit for the shell, such that the macromolecule remains nearly spherical. ${ }^{37}$ Case (c) is similar to (b), where the chains of a dendritic scaffold now also contain mesogens. The molecular architecture is sometimes referred to as "mainchain dendrimer." 38 In both cases, (b) and (c), the interior of the macromolecule is accessible to mesogens. Therefore, the soft colloid can be interpreted as a composite object formed by a dendritic scaffold, shell mesogens, and mesogens temporarily confined to its interior. All examples shown in Fig. 1 comport to homeotropic surface anchoring. In addition, a tangential anchoring scenario can be realised if the mesogens are attached laterally. ${ }^{37}$ Other experimentally realisable
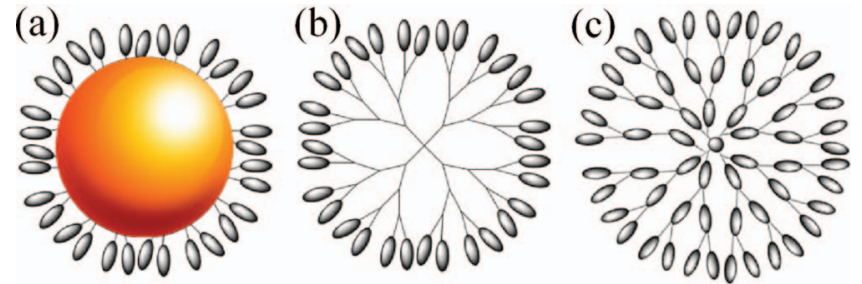

FIG. 1. Macromolecular realisations of spherical colloids with homeotropic surface anchoring: (a) solid nanoparticle with tethered chains containing mesogens; (b) carbosilane LC dendrimer with dense shell of terminal mesogens; (c) main-chain LC dendrimer. 
systems worth mentioning are: optically induced cholesteric toroidal droplets inside the nematic host, ${ }^{39}$ active elastomeric LC particles, ${ }^{40}$ and, possibly, some stable micellar structures.

Let us first consider mesoscopic models for soft colloids corresponding to cases (b) and (c) in Fig. 1. To this end the following simplifications will be made:

(i) the only important feature of the colloid taken into account is the existence of a spherical shell with oriented mesogens,

(ii) fluctuations of the shape of the colloid shape are neglected, and

(iii) the internal structure of the colloid is modelled implicitly.

One of the simplest realisations of this model is a spherical region of radius $R_{c}$ where an orienting field is applied inside a spherical shell. This approach bears conceptual similarity to the work of Tanaka and Araki, ${ }^{41}, 42$ where the colloid is modelled as a spherical region with higher viscosity compared to that of the bulk. In our case, the orienting field constrains rotational degrees of freedom of the mesogens. This effect can be interpreted as an effective increase of their rotational viscosity. The orienting field may serve to maintain homeotropic, tangential, or even tilted orientations of the mesogens in the shell with respect to the colloid's local surface normal. In our model of a soft colloid mesogens from the bulk are free to enter or leave the interior of the colloid similar to real LC molecules moving into or out from the interior of a LC dendrimer.

We consider three different realisations of the soft colloid distinguished by the thickness of a spherical shell in which the orienting field is applied. Taking $R_{c}$ to be the radius of the outer circumference of the soft colloid, an outer shell of thickness $\Delta R=R_{c} / 3$ resembles the carbosilane dendrimer with terminal mesogens and orientationally neutral colloid interior [see Fig. 1(b)]. The shell of thickness $\Delta R=R_{c}$ can be related to the main-chain LC dendrimer, where mesogens trapped inside a colloid are subject to a local radial field created by their counterparts from the dendritic scaffold [see Fig. 1(c)]. Finally, for reasons of comparison, we also consider an intermediate shell thickness of $\Delta R=R_{c} / 2$. We also note that the soft-colloid model with a shell thickness of $R_{c} / 3$ and $R_{c} / 2$ allows to study defects inside a spherical cavity with radial anchoring, a geometry that appears in the case of polymer dispersed LCs. ${ }^{43-45}$ The cases of shell thickness of $R_{c} / 3$ and $R_{c}$ are illustrated by snapshots in Fig. 2 .

Let us now turn to a model that mimics a decorated colloid [see Fig. 1(a)] immersed in a nematic LC host. In this case the interior of the colloid is modelled explicitly via a sphere of radius $R_{c}$ inaccessible to bulk mesogens. Here, $N_{\mathrm{sh}}$ shell mesogens are grafted onto the surface of this sphere. We consider the simplest case of quenched grafting, where the centers of $N_{\mathrm{sh}}$ mesogens are located on the surface of a central sphere. Throughout the simulation both positions and orientations of the grafted mesogens are quenched such that these mesogens are completely immobilized and define a certain anchoring pattern. In the case of a soft colloid we restrict the discussion to homeotropic and tangential (i.e., degenerate planar) anchoring only. By contrast, for a decorated col-
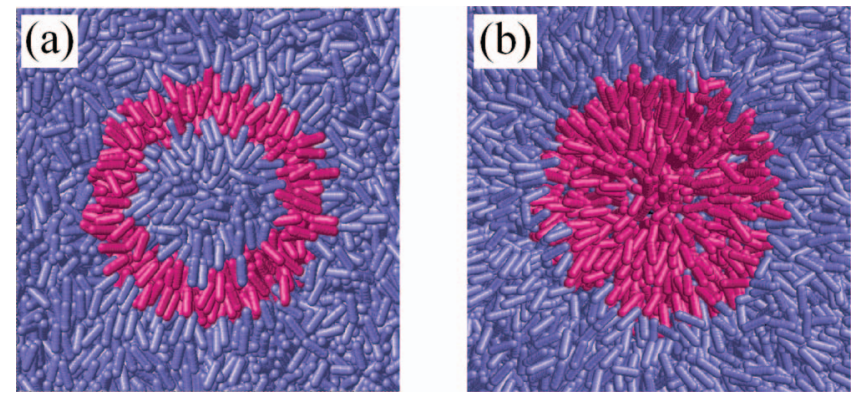

FIG. 2. Snapshots for the soft colloid model with shell thickness of $\Delta R=R_{c} / 3$ (a) and $\Delta R=R_{c}$ (b). Mesogens inside the shell are colored in pink, whereas mesogens outside the shell are colored in blue.

loid one can control the orientation of a single easy axis on the colloid's surface, by regular arrangement of the grafted mesogens. If this arrangement is made locally uniaxial planar anchoring is realised. Mapping of the homeotropical anchoring pattern onto a spherical surface is unambiguous, resulting in a spherically symmetric hedgehog-like metaparticle [see Fig. 3(a)]. On the contrary, mapping of a uniform planar pattern onto a sphere is ambiguous. It can be imagined as first folding a flat surface onto a cylinder and then mapping this folded pattern onto a spherical surface. The resulting anchoring pattern on a sphere will depend on the angle between the easy axis $\widehat{\mathbf{a}}$ and the symmetry axis of the cylinder $\widehat{\mathbf{c}}$. We restrict our study to two characteristic cases where the two are either collinear or orthogonal illustrated in Figs. 3(b) and 3(c), respectively. We refer to the situation depicted in Fig. 3(b) as planar azimuthal anchoring, whereas the one illustrated in Fig. 3(c) is referred to as longitudinal planar anchoring. To justify the term "planar anchoring" we restrict our study to a regular arrangement of mesogens on the surface mimicking the meridians and parallels, respectively, as shown in Fig. 3.

For simplicity we assume grafted mesogens to be the same as their bulk counterparts. Therefore, surface anchoring is maintained solely because of the interaction between

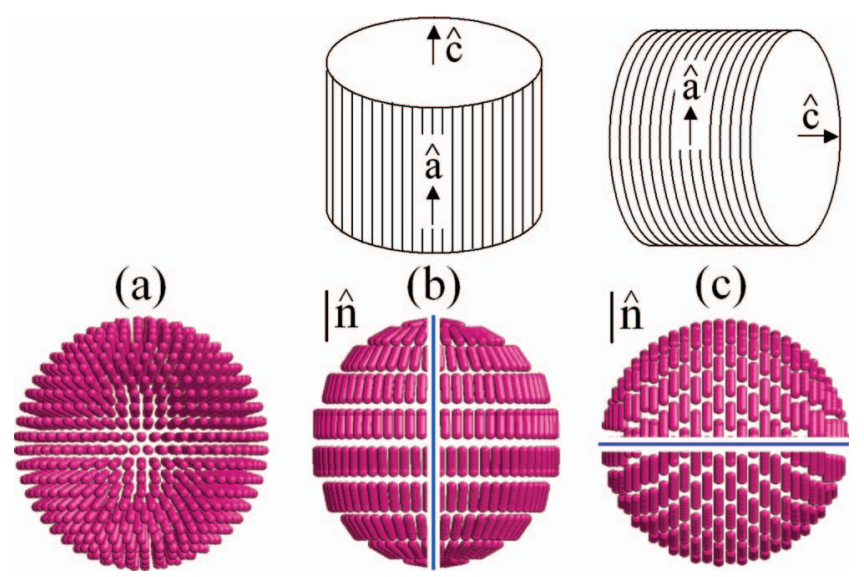

FIG. 3. Snapshots for the decorated colloid model with homeotropic (a), planar azimuthal (b), and planar longitudinal (c) anchoring. For the latter two cases cartoons on the top row of the figure illustrate the folding of a flat surface with planar anchoring onto a cylinder. The easy axis $\widehat{\mathbf{a}}$ in case (b) and the cylinder symmetry axis $\widehat{\mathbf{c}}$ are collinear. Both are orthogonal in case (c). In cases (b) and (c) and for the images on the bottom row of the figure the blue line indicates the direction of $\widehat{\mathbf{c}}$. Here the bulk director $\widehat{\mathbf{n}}$ is also shown. 

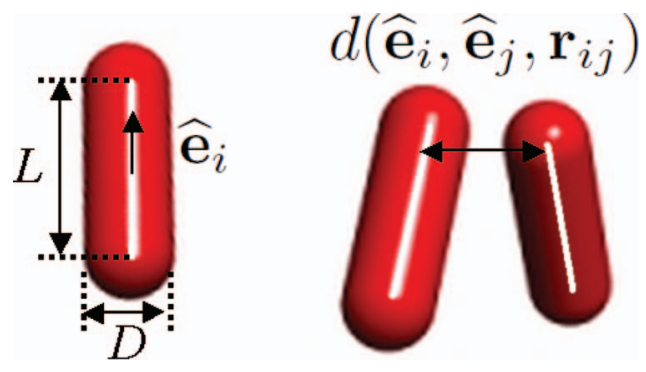

FIG. 4. Schematic representation of spherocylindrical particles of breadth $D$ and length $L$ and the minimal distance $d_{i j}$ between their cores.

grafted and bulk mesogens. In this case the anchoring strength is controlled solely by the grafting density. We defer a more detailed discussion of interaction potentials representing our prototypical cases to Secs. III and IV.

\section{PROPERTIES OF NEMATIC HOST PHASE}

Before we address the main topic of this study, namely, the perturbation of the director field around a spherical colloid in a nematic host phase, let us concentrate on properties of the host in the absence of a colloid. However, we remind the reader at this stage that we choose a mesoscale description so that individual mesogens correspond to molecular assemblies rather than individual molecules. Therefore, the precise shape of our mesogens is largely irrelevant.

The pair interaction potential used in this study has been developed by Lintuvuori and Wilson. ${ }^{29}$ Each spherocylindrical mesogen is assumed to have breadth $D$ and length $L$. The location of the $i$ th mesogen is determined by its center of mass position $\mathbf{r}_{i}$, whereas its orientation is given by a unit vector $\widehat{\mathbf{e}}_{i}$ pointing along its long axis (see Fig. 4). Following Kihara, ${ }^{46}$ repulsive interactions between the pair of mesogens depend on the minimum distance $d\left(\widehat{\mathbf{e}}_{i}, \widehat{\mathbf{e}}_{j}, \mathbf{r}_{i j}\right)$ between their cores. This distance is a function of the orientation of both mesogens $\widehat{\mathbf{e}}_{i}$ and $\widehat{\mathbf{e}}_{j}$, and of the distance vector $\mathbf{r}_{i j}$ connecting their centers. For notational convenience we introduce shorthand notation $\mathbf{q}_{i j}=\left\{\widehat{\mathbf{e}}_{i}, \widehat{\mathbf{e}}_{j}, \mathbf{r}_{i j}\right\}$ to refer to this set of variables. Because of the mesoscale nature of our model we adopt a quadratic form $U\left[1-d^{\prime}\left(\mathbf{q}_{i j}\right)\right]^{2}$ for the repulsive part of the interaction potential, where $d^{\prime}\left(\mathbf{q}_{i j}\right)=d\left(\mathbf{q}_{i j}\right) / D$ and $U$ sets the energy scale of the repulsion.

In the following we will use dimensionless quantities and define length and energy in units of $D$ and $U$, respectively, where we use the prime to indicate dimensionless quantities throughout this work. Other dimensionless quantities are introduced via suitable combinations of these basic units. For example, dimensionless density may be expressed as $\rho^{\prime}$ $=\rho D^{3} / m$, where $m$ is the mass of a mesogen. Similarly, dimensionless temperature is given as $T^{\prime}=k_{\mathrm{B}} T / U$, dimensionless pressure is introduced through $P^{\prime}=P D^{3} / U$, and dimensionless time may be expressed as $t^{\prime}=t\left(m D^{2} / U\right)^{-1 / 2}$. In all these expressions unprimed quantities are given in SI units.

We note in passing that quadratic form for soft repulsion originated from dissipative particle dynamics, ${ }^{47-49}$ which is a mesoscopic simulation technique for spherical bodies. The softness of this potential is reflected by the fact that the maximum energy at complete overlap of two cores is finite. The purely repulsive model for the spherocylinders, however, is prone to insufficient nematogenicity ${ }^{50-52}$ as a result of both weak stericity and lack of anisotropic attractive terms.

Lintuvuori and Wilson ${ }^{29}$ have therefore complemented this soft repulsive potential by an attractive part, similar in spirit to the earlier work by Steuer et al. ${ }^{53}$ Here, the repulsive part of the potential is shifted downwards in the interval of $0 \leq d^{\prime}\left(\mathbf{q}_{i j}\right) \leq 1$ by a configuration dependent well depth $\epsilon\left(\mathbf{q}_{i j}\right)$. For $1 \leq d^{\prime}\left(\mathbf{q}_{i j}\right) \leq d_{c}^{\prime}$ an additional orientation dependent attractive term is added to the potential which is cut off if $d^{\prime}\left(\mathbf{q}_{i j}\right)$ exceeds the (dimensionless) cutoff distance $d_{c}^{\prime}$. Specifically, we take the interaction potential to be given by the expression

$U_{i j}\left(\mathbf{q}_{i j}\right)=\left\{\begin{array}{ll}U\left\{\left[1-d^{\prime}\left(\mathbf{q}_{i j}\right)\right]^{2}-\epsilon^{\prime}\left(\mathbf{q}_{i j}\right)\right\}, & d^{\prime}\left(\mathbf{q}_{i j}\right)<1, \\ U\left\{\left[1-d^{\prime}\left(\mathbf{q}_{i j}\right)\right]^{2}-\epsilon^{\prime}\left(\mathbf{q}_{i j}\right)\right. & \\ \left.-\frac{1}{4 \epsilon^{\prime}\left(\mathbf{q}_{i j}\right)}\left[1-d^{\prime}\left(\mathbf{q}_{i j}\right)\right]^{4}\right\}, & d^{\prime}\left(\mathbf{q}_{i j}\right) \in\left[1, d_{c}^{\prime}\right] \\ 0, & d^{\prime}\left(\mathbf{q}_{i j}\right)>d_{c}^{\prime}\end{array}\right.$,

where the dimensionless configuration dependent well depth $\epsilon^{\prime}\left(\mathbf{q}_{i j}\right)$ can be obtained from the requirement that both the potential and its first derivative vanish at $d_{c}^{\prime}$. This gives ${ }^{29}$

$$
\epsilon^{\prime}\left(\mathbf{q}_{i j}\right)=\left\{4\left[U_{a}^{\prime}-5 \epsilon_{1}^{\prime} P_{2}\left(\widehat{\mathbf{e}}_{i} \cdot \widehat{\mathbf{e}}_{j}\right)\right]\right\}^{-1},
$$

where $U_{a}^{\prime}$ and $\epsilon_{1}^{\prime}$ are the dimensionless counterparts of the parameters $U_{\text {attr }}^{*}$ and $\epsilon_{1}$, respectively, introduced in Ref. 29, and $P_{2}(x)=\left(3 x^{2}-1\right) / 2$ is the second Legendre polynomial.

Following Ref. 29 we take $U_{a}^{\prime}=6, \epsilon_{1}^{\prime}=12 / 25$ and $L^{\prime}=3$. For the side-side and end-end configuration of a mesogenic pair the interaction potential is illustrated by the plots in Fig. 5. Because of the equal well depths for both configurations a formation of smectic phases is suppressed.

The phase diagram of this model has been studied in Ref. 29 for a broad range of parameters. For the current choice of parameters it has also been shown in Ref. 29 that the phase diagram includes a nematic phase (see Figure 4 of Ref. 29). We conducted molecular dynamics simulations using a cubic box with linear system size of $X^{\prime}=53.5$ together with periodic boundary conditions. The simulations are carried out in the canonical ensemble with a timestep of $\Delta t^{\prime}=0.0426$.

For $\rho^{\prime}$ less than 0.16 we observe micro-phase separated nematic droplets coexisting with isotropic regions. For $\rho^{\prime} \geq 0.2(N=30462$ mesogens) a stable nematic phase is observed over the range of temperatures $0.047 \leq T^{\prime} \leq 0.055$. We

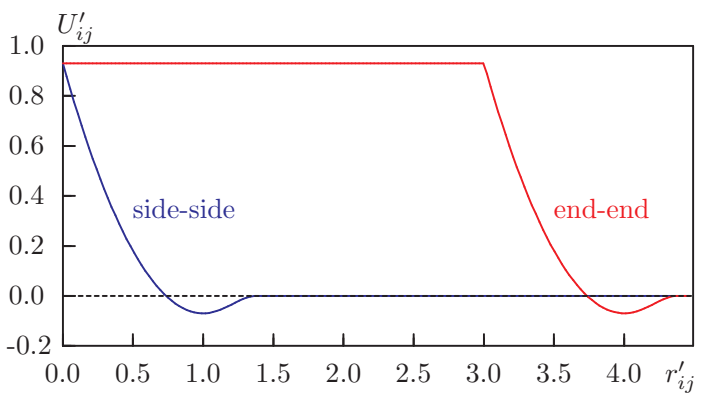

FIG. 5. Pair potential $U_{i j}$ [see Eq. (1)], in reduced units. Side-side and endend arrangement of spherocylinders are shown only, indicated in the figure. $r_{i j}$ is the distance between the centers of the spherocylinders. 


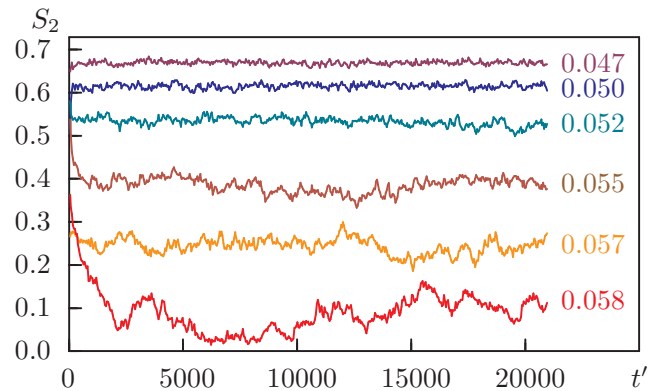

FIG. 6. Evolution of the nematic order parameter $S_{2}$ for a series of heating runs at reduced density of $\rho^{\prime}=0.2$ for $N=30462$ particles. Temperature $T^{\prime}$ is indicated in the plot.

characterise the nematic phase through the order parameter

$$
S_{2}=\left\langle P_{2}\left(\widehat{\mathbf{e}}_{i} \cdot \widehat{\mathbf{n}}\right)\right\rangle \text {, }
$$

where $\widehat{\mathbf{n}}$ is the nematic director and averaging is performed over all mesogens. Plots in Fig. 6 illustrate the evolution of $S_{2}$ as time progresses and for a sequence of temperatures as indicated in the figure. Starting from the lowest $T^{\prime}$, runs at higher $T^{\prime}$ always start from the equilibrated last configuration of the immediately preceding lower $T^{\prime}$. As one can see from Fig. $6, S_{2} \approx 0.67$ at $T^{\prime}=0.047$, whereas the smectic order parameter (not shown) is of the order of $10^{-2}$.

This confirms that at $T^{\prime}=0.047$ a stable nematic phase exists. As expected, $S_{2}$ decreases with increasing $T^{\prime}$. Moreover, the plots in Fig. 6 indicate that fluctuations of $S_{2}$ increase as one approaches the stability limit of the nematic phase from below. Up to $T^{\prime}=0.056$ the system remains uniform. At higher temperatures domains are forming characterised by locally different directors. Consequently, at temperatures $T^{\prime} \gtrsim 0.056$ the phase is globally isotropic.

Let us concentrate now on properties of the model LC system in the range $0.047 \leq T^{\prime} \leq 0.058$ and $\rho^{\prime}=0.2$. Focusing on its elastic properties first these can be analysed via the Frank elastic constants $K_{1}, K_{2}$, and $K_{3}$. Following Refs. 54 and 55 these material constants can be calculated from the Fourier transform

$$
\widehat{\mathbf{Q}}(\mathbf{k})=\int \mathbf{Q}(\mathbf{r}) \exp (i \mathbf{k} \cdot \mathbf{r}) d \mathbf{r}
$$

of the order tensor

$$
\mathbf{Q}(\mathbf{r})=\frac{1}{2 \rho(\mathbf{r})} \sum_{i=1}^{N}\left\langle\left[3 \widehat{\mathbf{e}}_{i} \otimes \widehat{\mathbf{e}}_{i}-\mathbf{1}\right] \delta\left(\mathbf{r}-\mathbf{r}_{i}\right)\right\rangle,
$$

where $\rho(\mathbf{r})$ is the local density, the operator $\otimes$ indicates the tensor product, $\mathbf{1}$ is the unit tensor, and $\delta$ is the Dirac $\delta$ function. We define a Cartesian coordinate system a, b, c such that $\mathbf{Q}(\mathbf{r})$ is diagonal and the local director is $\widehat{\mathbf{n}}(\mathbf{r})=(0,0,1)$. For a wave vector $\mathbf{k}=\left(k_{1}, 0, k_{3}\right)$ in the $1-3$ plane we introduce

$$
\begin{aligned}
& W_{13}\left(k_{1}, k_{3}\right)=\frac{9}{4} \frac{S_{2}^{2} V k_{\mathrm{B}} T}{\left\langle\left|\widehat{Q}_{13}(\mathbf{k})\right|^{2}\right\rangle}=K_{1} k_{1}^{2}+K_{3} k_{3}^{2}, \\
& W_{23}\left(k_{2}, k_{3}\right)=\frac{9}{4} \frac{S_{2}^{2} V k_{\mathrm{B}} T}{\left\langle\left|\widehat{Q}_{23}(\mathbf{k})\right|^{2}\right\rangle}=K_{2} k_{1}^{2}+K_{3} k_{3}^{2},
\end{aligned}
$$

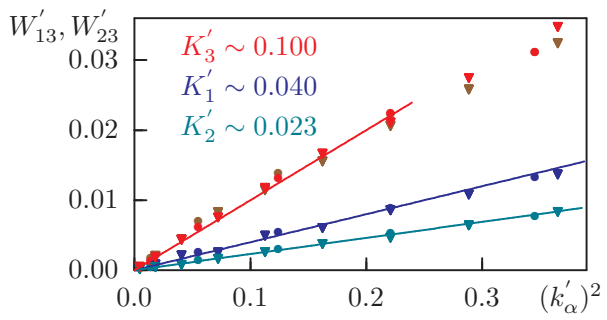

FIG. 7. Dependence of the functions $W_{13}^{\prime}$ and $W_{23}^{\prime}$ on the square of a relevant non-zero argument [see Eqs. (6) and (7)] denoted as $k_{\alpha}^{\prime 2}$, at $T^{\prime}=0.050$. Linear system sizes are: $X^{\prime}=54$ (circles) and 95 (triangles). All data data related to each of $K_{1}^{\prime}, K_{2}^{\prime}$, and $K_{3}^{\prime}$ are grouped into three respective sets. The values obtained for elastic constants via linear fits are shown in the top left corner.

in the limit of $k \rightarrow 0$. Plotting $W_{13}$ and $W_{23}$ as functions of $k_{1}^{2}$ and $k_{3}^{2}$, respectively, we obtain $K_{1}, K_{2}$, or $K_{3}$ from linear fits to the discrete data points.

To check the system size dependence of the elastic constants we perform simulations at $\rho^{\prime}=0.2$ and $T^{\prime}=0.050$ for two system sizes with linear dimensions of $X^{\prime}=54$ and 95. All runs are started from a perfectly aligned state and the system was allowed to equilibrate for $t^{\prime}=4500$ time units. Unlike Refs. 54 and 55 we do not use dynamics with a constrained director, but the latter is free to fluctuate. A set of configurations in the equilibrium state is considered for averaging, separated by 500 time steps. For each configuration an instant global nematic director is found first and then the positions and orientations of all mesogens are rotated into the director frame. Evaluation of $K_{1}^{\prime}, K_{2}^{\prime}$, and $K_{3}^{\prime}$ is performed in the director frame. In Fig. 7 we plot $W_{13}^{\prime}$ and $W_{23}^{\prime}$ as functions of $k_{\alpha}^{\prime 2}(\alpha=1,2,3)$. For the case of $K_{3}^{\prime}$ we used both the estimates from $W_{13}^{\prime}\left(0, k_{3}^{\prime}\right)$ and $W_{23}^{\prime}\left(0, k_{3}^{\prime}\right)$ grouped together. As one can see from Fig. 7, the slopes for both system sizes essentially coincide for sufficiently small values of the wave vector. Moreover, data points for $W_{13}^{\prime}$ and $W_{23}^{\prime}$ are identical to three significant digits. Repeating the above analysis for different temperatures in Fig. 8 indicates that all three elastic constants vanish at the transition from the nematic to the isotropic phase according to one's intuition.

From the temperature dependence of the elastic constants plotted in Fig. 8 we are now in a position to establish the energy scale pertinent to our model. To reach this goal we assume our coarse-grained LC to represent para-Azoxyanisole (PAA) for which the temperature dependence of ratios of the elastic constants have been measured experimentally. ${ }^{56,57}$ The

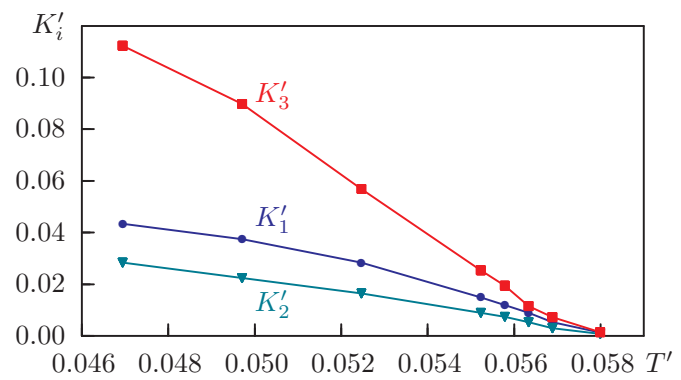

FIG. 8. Temperature dependence of the elastic constants $K_{1}^{\prime}, K_{2}^{\prime}$, and $K_{3}^{\prime}$ on reduced temperature $T^{\prime}$. 


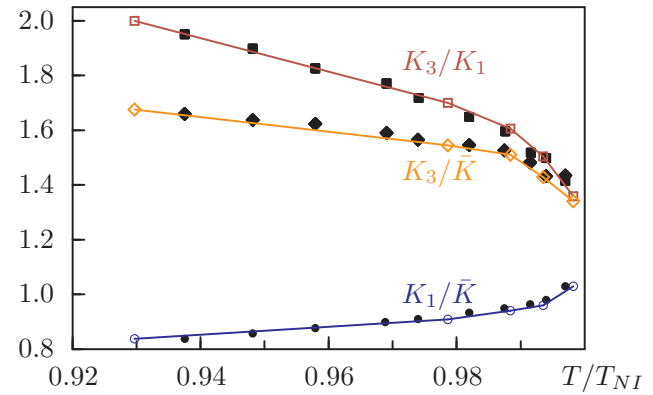

FIG. 9. Temperature dependence of the ratios $K_{3} / K_{1}, K_{1} / \bar{K}$, and $K_{3} / \bar{K}$ of elastic constants $\left[\bar{K}=\left(K_{1}+K_{2}+K_{3}\right) / 3\right]$ near the nematic-isotropic transition point $T_{\mathrm{NI}}$. Black solid symbols are experimental data for PAA ${ }^{56,57}\left(T_{\mathrm{NI}}\right.$ $=422 \mathrm{~K}$ ). Open symbols are simulation data obtained in this work. Solid lines are intended to guide the eye.

temperature variation of these ratios is presented in Fig. 9 for both the experimental data as well as for our coarse-grained model. For our model system we adjusted the temperature of the isotropic-nematic phase transition $T_{\mathrm{NI}}$ so that an optimal agreement between both data sets is achieved. Comparing $T_{\mathrm{NI}}^{\prime}$ for the coarse-grained model with the experimental value $T_{\mathrm{NI}}=422 \mathrm{~K}$ for PAA we estimate the energy scale to be characterised by $U \sim 10^{-19} \mathrm{~J}$. This number seems reasonable in view of the strength of typical force fields used in the simulation of hydrocarbons. ${ }^{58}$

To identify the length-scale of our model we analyse the mesogen-mesogen pair orientation correlation function ${ }^{59}$

$$
G_{2}(r)=\left\langle P_{2}\left[\widehat{\mathbf{e}}_{i}\left(\mathbf{r}_{i}\right) \cdot \widehat{\mathbf{e}}_{j}\left(\mathbf{r}_{j}\right)\right]\right\rangle,
$$

where $r=\left|\mathbf{r}_{i}-\mathbf{r}_{j}\right|$ is the distance between mesogens $i$ and $j$ located at $\mathbf{r}_{i}$ and $\mathbf{r}_{j}$, respectively. The averaging is performed over all pairs located in a thin shell of width $\Delta r^{\prime} \approx 0.5$ separated by a distance $r$ from reference mesogen $i$. In a spin system a similar correlation function can be defined which has the same asymptotic behavior. In the context of this study, however, it is more convenient to consider the connected orientation correlation function

$$
G_{2}^{c}(r)=G_{2}(r)-S_{2}^{2} \stackrel{r \rightarrow \infty}{=} \frac{A}{r^{a}} \exp \left[-r / \xi_{N}\right],
$$

where $A$ is a constant, $\xi_{N}$ is the nematic coherence (correlation) length and $a$ is some exponent. A semi-logarithmic plot of $G_{2}^{c}(r)$ for $\rho^{\prime}=0.2$ and $T^{\prime}=0.050$ is presented in Fig. 10 . The best fit is achieved at $a \approx 0.75$ and the estimate for the nematic coherence length is $\xi_{N}^{\prime} \approx 10.2$. Typical experimental values for this quantity are of the order of $10 \mathrm{~nm} .{ }^{60}$ Together with this information our fitted value allows us to estimate

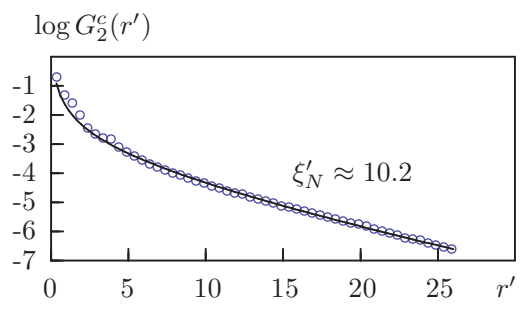

FIG. 10. Plot of $\log G_{2}^{c}\left(r^{\prime}\right)$ as a function of $r^{\prime}$ (see text for more details).
$D \sim 10 \AA$. This is a reasonable value for the coarse-grained particle corresponding to a cluster of 7-10 LC molecules.

\section{SOFT COLLOID}

Let us now turn to a discussion of defects evolving around a soft colloid introduced in Sec. II. Mesogens located inside a shell of thickness $\Delta R$ are subjected to an external potential

$$
U_{\mathrm{ex}}^{\prime}\left(\widehat{\mathbf{e}}_{i}, \widehat{\mathbf{s}}_{i}\right)= \begin{cases}-f_{c}^{\prime}\left(\widehat{\mathbf{e}}_{i} \cdot \widehat{\mathbf{s}}_{i}\right)^{2}, & R_{c}^{\prime}-\Delta R^{\prime}<s_{i}^{\prime}<R_{c}^{\prime}, \\ 0, & s_{i}^{\prime} \leq R_{c}^{\prime}-\Delta R^{\prime}, s_{i}^{\prime} \geq R_{c}^{\prime},\end{cases}
$$

which serves to orient mesogens in that shell. In Eq. (10), $\mathbf{s}_{i}^{\prime}=\mathbf{r}_{i}^{\prime}-\mathbf{R}^{\prime}$, where $\mathbf{R}^{\prime}$ is the position of the center of the colloid and all quantities are given in reduced units introduced in Sec. III. The quantity $f_{c}^{\prime}$ on the first line of Eq. (10) allows us to control the alignment of mesogen $i$ in respect to the local surface normal of the colloid. In particular, for $f_{c}^{\prime}>0$, local homeotropic alignment of mesogens within the shell is realised, whereas for $f_{c}^{\prime}<0$ locally tangential orientation is favored. Regardless of the sign of $f_{c}^{\prime}$ it is important to restrict the range of values of this quantity such that $\left|f_{c}^{\prime}\right| \leq T^{\prime}$. If this relation is violated it turned out in practice that thermostatting the system can lead to artifacts.

Throughout this section we fix $\rho^{\prime}=0.2$ and $T^{\prime}=0.050$, a state point in the lower left corner of the phase diagram presented in Fig. 4 of the work Lintuvuori and Wilson, ${ }^{29}$ where the bulk host phase is nematic. To make contact with the different parametrisation used in this earlier work, we note that our state point corresponds to $\rho^{*}=0.2$ and $T^{*}=1.25$ in Ref. 29.

Because the outer surface of the shell is only imaginary, we monitored the rate at which it was crossed by mesogens both in the in- and outward direction during the course of the simulation. For $\Delta R^{\prime}=R_{c}^{\prime}=13.4,0.1 \%$ of mesogens per timestep are crossing the outer shell boundary in each direction on average. Therefore, an exchange between mesogens inside our soft colloid and the surrounding bulk is considered irrelevant.

We begin with the case of homeotropic anchoring realised by $f_{c}^{\prime}>0$. Taking the size of our colloid $R_{c}^{\prime}=13.4$ and the value of the nematic coherence length, as determined in Sec. III, gives $R_{c} / \xi_{N} \approx 1.3$, for which one would expect the Saturn ring defect topology to develop. ${ }^{10}$ In SI units and based upon our estimate of the coherence length, the diameter of our colloid corresponds to about $27 \mathrm{~nm}$.

We performed simulations starting from different initial configurations such as: uniaxial, random, and a configuration which has radial symmetry with respect to the center of the box. All these initial configurations lead to the same equilibrium structures inside and outside the colloid. During equilibration the system relaxes to a thermodynamic state in which the radial orientation of the mesogens is maintained only within the region where the external potential $U_{\text {ex }}$ acts. Outside that region mesogens self-assemble into a nematic phase characterized by an uniaxial director field. Once the simulation has completed we analyze the structure of the equilibrium phase using a set of configurations separated by 10000 
(a)

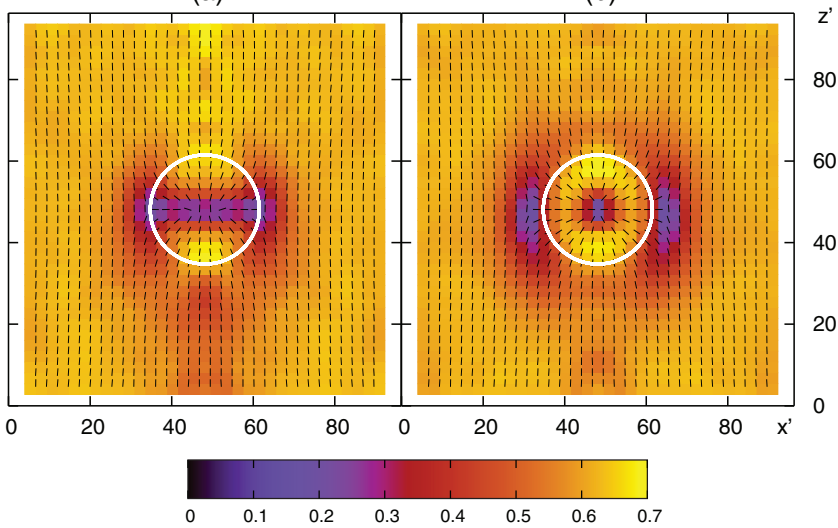

FIG. 11. Plots of the local director field and the local nematic order parameter in the $\mathrm{x}-\mathrm{z}$ plane. The attached color bar specifies the magnitude of the local nematic order parameter, whereas dashes represent the direction of the local nematic director. In both cases $R_{c}^{\prime}=13.4$ as indicated by the white circle. (a) $f_{c}^{\prime}=0.20$, (b) $f_{c}^{\prime}=0.33$. In both cases $T^{\prime}=0.050$ and $X^{\prime}=94$.

time steps. For each configuration the global director is determined first. To that end we solve an eigenvalue equation for the non-local analogue of $\mathbf{Q}(\mathbf{r})$ in Eq. (5), where we take into account all mesogens of which our system is composed. After this global nematic director has been determined we take the $\mathrm{z}$-axis to be collinear with it and transform all the positions and orientations into the director frame. In this frame we then compute the local nematic order parameter and the local director field. The final results are obtained by averaging these local properties over the set of configurations. Because of the cylindrical symmetry of the system both local director field and nematic order parameter can be represented by a twodimensional (2D) map.

Plots of these maps in Fig. 11 have been obtained for two different values of $f_{c}^{\prime}$. The plot in Fig. 11(a) reveals that the defect is more or less located on the surface of the colloid. Because of our 2D representation regions of low nematic order (the "defect") actually form a line coming out and going into the paper plane. This defect topology is know as a surface ring. ${ }^{10,25}$ At larger $f_{c}^{\prime} \geq 0.2$, the order inside the colloid increases and therefore the mismatch between the nematic director outside the colloid and inside arises at a somewhat larger distance from the colloid's center. This topology has been termed Saturn ring. Similar effects have been observed by Andrienko et $a l^{23}$ and Melle et $a .^{21}$ In both studies molecule-based models were considered as opposed to the soft coarse-grained one employed in this study.

Because the plots in Fig. 11 already show that regions of low nematic order can be displaced away from the colloid surface by increasing $f_{c}^{\prime}$, a more detailed investigation of the impact of $f_{c}^{\prime}$ seems worthwhile. A suitable way to find the position $R_{d}$ of the defect line relative to the colloid center is to search for the minimum local nematic order. Plots in Fig. 12 indicate the variation of $R_{d}^{\prime}$ with increasing $f_{c}^{\prime}$. From the figure it is evident that in the case where an external field is applied everywhere inside the colloid, $R_{d}^{\prime}$ increases monotonically with $f_{c}^{\prime}$ but exhibits a tendency to level off if $f_{c}^{\prime}$ is sufficiently large. Similar behavior is observed if the external field is applied in a shell of thickness $\Delta R=R_{c} / 2$ and $\Delta R=R_{c} / 3$.

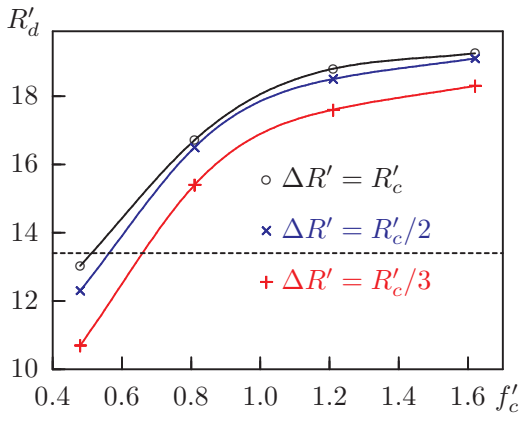

FIG. 12. Position $R_{d}^{\prime}$ of the Saturn ring relative to the center of a colloid as a function of the strength $f_{c}^{\prime}$ of the external potential and variable thickness indicated in the figure. For comparison the value $R_{d}^{\prime}=R_{c}^{\prime}$ is indicated by horizontal dashed line.

Not surprisingly, $R_{d}^{\prime}$ at any given value of $f_{c}^{\prime}$ is smallest for the smallest shell thickness considered in Fig. 11. Between $\Delta R=R_{c}$ and $\Delta R=R_{c} / 2$ very little difference is detected. This increase of $R_{d}^{\prime}$ with $f_{c}^{\prime}$ irrespective of the shell thickness can be interpreted as an increase of the effective size of the colloid. The exact size cannot be defined precisely given the coarse-grained (i.e., soft) nature of the model colloid.

Notice also from plots in Fig. 11 that besides regions of low nematic order outside the colloid similar regions exist on the inside. This is to be expected because the uniaxial director field is in conflict with the radial symmetry of the external potential. This situation is similar to the one encountered in polymer-dispersed LCs with radial anchoring. ${ }^{43-45}$ Therefore, the case of finite shell thickness allows one to study both external and internal defects. A more detailed insight into a dependence of internal and external disclination rings on the thickness of the shell is provided by plots presented in Fig. 13. These plots have been obtained by fixing the strength (a)

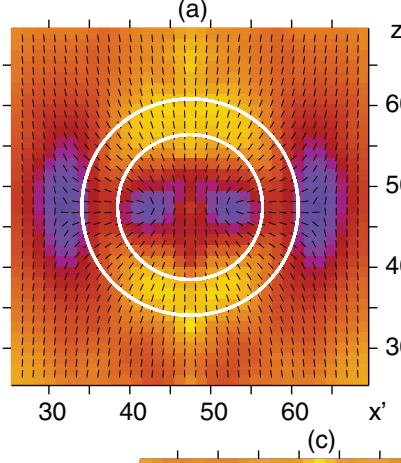

(b)
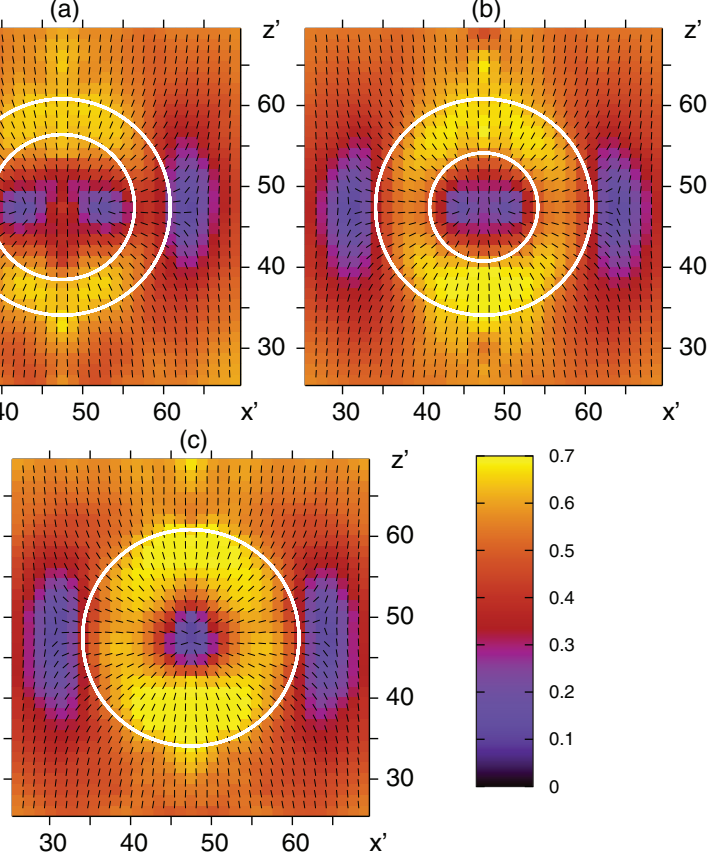

FIG. 13. As Fig. 11, but for $f_{c}^{\prime}=0.33$ and (a) $\Delta R^{\prime}=R_{c}^{\prime} / 3$, (b) $\Delta R^{\prime}=R_{c}^{\prime} / 2$, and (c) $\Delta R^{\prime}=R_{c}^{\prime}$. White rings indicate the size of the shell in which $U_{\text {ex }}$ [see Eq. (10)] is applied. 
of the external potential and the temperature. As one can see by comparing plots in Fig. 13, the position of the outer disclination ring remains roughly constant with respect to the colloid's surface as the shell thickness increases. The position of the inner disclination ring relative to the inner surface of the sperical shell remains also roughly constant such that the radius of the internal disclination ring shrinks with increasing shell thickness and eventually vanishes for the case of $\Delta R$ $=R_{c}$. One can clearly see the internal ring defect ${ }^{43,45}$ in the cases (a) and (b), which reduces to a point defect in the case (c).

To study the impact of anchoring strength at the colloid's surface we consider the coherence length $\xi_{R}$ for surface anchoring. This quantity can be estimated similar to $\xi_{N}$, introduced in Eq. (9). More specifically, we consider the mesogencolloid pair correlation function

$$
R_{2}(s)=\left\langle P_{2}\left(\widehat{\mathbf{e}_{i}} \cdot \widehat{\mathbf{s}_{i}}\right)\right\rangle
$$

where $\mathbf{s}_{i}$ is introduced in Eq. (10). At large separations $s$, this function tends to $S_{R}^{2}$, a square of the radial order parameter $S_{R}$, defined similarly to the nematic order parameter $S_{2}$ [see Eq. (3)], namely,

$$
S_{R}=\left\langle P_{2}\left(\widehat{\mathbf{e}}_{i} \cdot \widehat{\mathbf{s}}_{i}\right)\right\rangle,
$$

where averaging is performed over all mesogens. Assuming an uniaxial nematic phase, it is easy to show that for the infinite system $S_{R}=0$. For finite-size systems, however, small but non-zero values of the order $S_{R} \sim 0.1$ are found. Therefore, the connected mesogen-colloid pair correlation function $R_{2}^{c}(s)$ can be considered for the finite-size system with the following asymptotics

$$
R_{2}^{c}(s)=R_{2}(s)-S_{R}^{2} \stackrel{s \rightarrow \infty}{=} \frac{B}{s^{b}} \exp \left[-s / \xi_{R}\right],
$$

where $B$ is a constant, $b$ is some exponent and $\xi_{R}$ is the radial coherence length.

A quantitative measure of the anchoring strength is the extrapolation length $\xi_{e}=K_{3} /\left(W R_{c}\right)$, where $W$ is the anchoring energy. ${ }^{10}$ From this expression it is obvious that $\xi_{e}$ is small in the strong anchoring limit and large in the weak anchoring regime. Unfortunately, in our case, we do not know $W$. However, it seems plausible to assume that $\xi_{e}$ is proportional to the ratio $\xi_{N} / \xi_{R}$. Hence, we anticipate $\xi_{R}$ to be larger than $\xi_{N}$ in the strong and smaller in the weak anchoring regime.

Plots in Fig. 14 show that with increasing $s, R_{2}^{c}(s)$ passes through a maximum at small values of $s$ and decays according to the far right side of Eq. (13) for sufficiently large $s$. By fitting the far right side of Eq. (13) to the data presented in Fig. 14 one can reliably extract the radial coherence length $\xi_{R}$. It is important to note that the slope for $R_{2}^{c}(s)$ at $s>R_{c}$ is the same for all the values of $f_{c}^{\prime}$ [see Fig. 14(a)] and for all shell thicknesses [the same figure (b)]. Within numerical accuracy of the fits we obtain $\xi_{R}^{\prime} \sim 4.5$ (the best fits are achieved at rather small exponents $0.1<b<0.2$ ), which is an average over the values obtained in all cases shown in Fig. 14. Comparing this number to $\xi_{N}^{\prime}$ (see Fig. 10) we notice that both are of the same order of magnitude and that $\xi_{e}$ is approximately constant. Because $\xi_{e}$ is inversely proportional to the anchoring strength, this implies that the latter cannot be modified
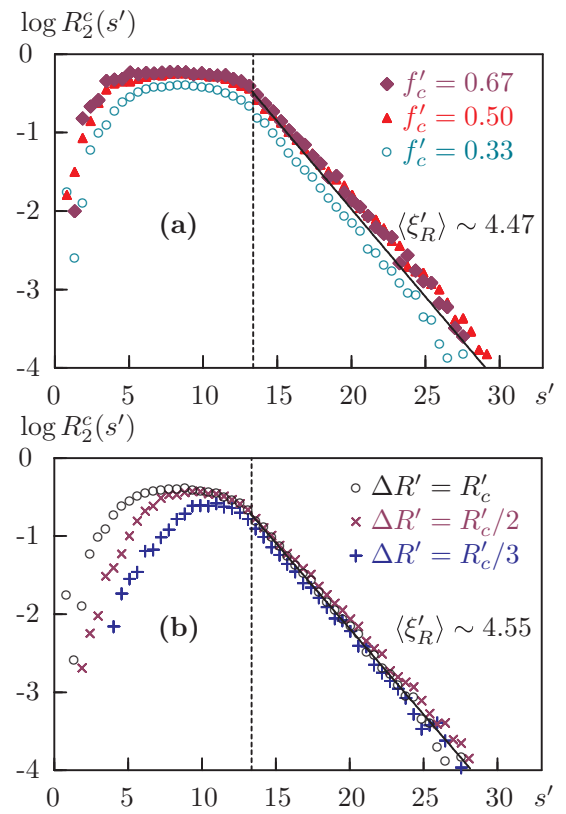

FIG. 14. Semi-logarithmic plot of the correlation function $R_{2}^{c}\left(s^{\prime}\right)$. (a) $\Delta R=$ $R_{c}$ and for various values of $f_{c}^{\prime}$ indicated in the figure; (b) for $f_{c}^{\prime}=0.33$ and various values of $\Delta R$ also indicated in the figure. Dashed vertical line in (a) and (b) marks the value of $R_{c}^{\prime}$.

by varying $f_{c}^{\prime}$ in Eq. (10). The apparent insensitivity of the anchoring strength upon a variation of $f_{c}^{\prime}$ prompts us to conclude that the anchoring strength is largely controlled by the density of the mesogens at colloid's surface, which remains approximately constant even though $f_{c}^{\prime}$ is varied significantly.

Turning next to a soft colloid with tangential anchoring we restrict the discussion to the same system size, density, and temperature as for its homeotropic counterpart presented above. In addition, only $\Delta R=R_{c}$ is considered here. To achieve tangential anchoring, $f_{c}^{\prime}<0$ is used in Eq. (10). As before for the homeotropically anchoring colloid, several starting configurations were considered without any effect on the final results.

In Fig. 15 we present plots of the local order parameter for a soft colloid with locally tangential anchoring of the mesogens. From Fig. 15(a) we notice that regions of low

(a)

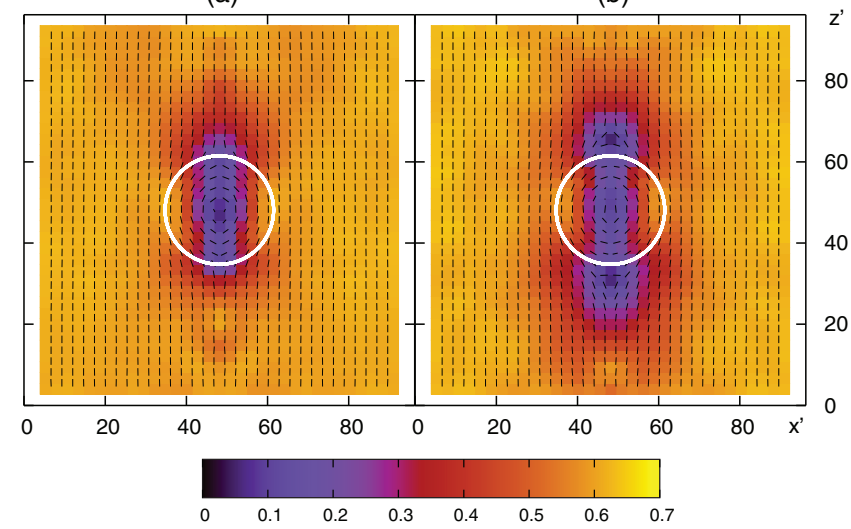

FIG. 15. As Fig. 11, but for tangential anchoring; (a) $f_{c}^{\prime}=-0.33$ and (b) $f_{c}^{\prime}=-0.5$. 


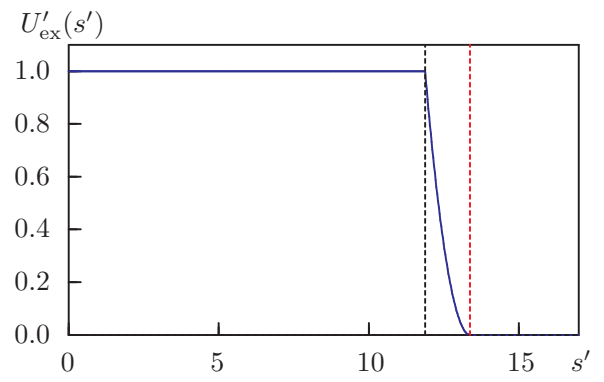

FIG. 16. External potential $U_{\mathrm{ex}}^{\prime}\left(s^{\prime}\right)$, given in Eq. (14) as a function of distance $s^{\prime}$ between the center of mass of a bulk mesogen from the center of the colloid.

nematic order exist outside the colloid centered on its north and south pole, respectively. This defect topology is reminiscent of a boojum defect. ${ }^{21,25,26}$ However, as one can see from Fig. 15(a), regions of low nematic order outside the colloid extend also into its interior. Therefore, the entire defect topology is not that of a boojum defect despite the tangential anchoring of the mesogens. Clearly, this is a consequence of the softness of the colloid. If one increases the magnitude of $f_{c}^{\prime}$, one can see from Fig. 15(b) that the overall defect topology does not change but the size of the region of low nematic order appears to be stretched along the z-axis in comparison with its counterpart in Fig. 15(a). Notice another subtle difference between the plots in Figs. 15(a) and 15(b). Whereas the minimum of local nematic order outside of the colloid is almost located on the north and south pole of the colloid in Fig. 15(a), these minima are moved away from the colloid's surface in Fig. 15(b). This is very much akin of the plots in Fig. 11 for the case of homeotropic anchoring.

\section{DECORATED COLLOID}

As we demonstrated in Sec. IV, for the case of a soft colloidal particle, varying the strength of the external potential does not change the anchoring strength but affects only the effective size of the colloid. This is because the density of the mesogens at the colloid's surface is equal to the bulk density. In order to get a handle on the anchoring strength, the colloid needs to be equipped with some sort of a surface with controllable density. From the perspective of our model, this implies that mesogens attempting to enter the interior of the colloid must feel a repulsive potential. Therefore, we replace $U_{\mathrm{ex}}^{\prime}$ in Eq. (10) by

$$
U_{\mathrm{ex}}^{\prime}\left(s_{i}^{\prime}\right)= \begin{cases}1, & s_{i}^{\prime}<R_{c}^{\prime}-L^{\prime} / 2 \\ \left(1-s_{i}^{\prime}\right)^{2}, & R_{c}^{\prime}-L^{\prime} / 2<s_{i}^{\prime}<R_{c}^{\prime}, \\ 0, & s_{i}^{\prime}>R_{c}^{\prime} .\end{cases}
$$

where $s_{i}^{\prime}$ has already been defined immediately after Eq. (10). Notice that $U_{\mathrm{ex}}^{\prime}\left(s_{i}\right)$ increases quadratically with decreasing $s_{i}^{\prime}$ in a spherical shell of thickness $L^{\prime} / 2$ and then becomes constant for all $s_{i}^{\prime}$ less then the inner boundary of this spherical shell. This potential is illustrated by the plot in Fig. 16.

This form of the potential can be rationalised as follows. As one can see from Eq. (1), the mesogen-mesogen interaction depends quadratically on the nearest distance between them. The lengthscale turns out to be set by $D$. Hence, a similar distance dependence has been adopted in Eq. (14) which mimics a spherical shell consisting of particles similar in size to the mesogens themselves. Finally, we realise from Eq. (14) that $U_{\mathrm{ex}}^{\prime}\left(s_{i}^{\prime}\right)$ does not depend on the orientation $\widehat{\mathbf{e}}_{i}$ of the mesogen. Hence, the colloid itself cannot control the orientation of a mesogen at its surface. This is achieved by grafting a shell of mesogens onto the repulsive spherical shell of the colloid. Grafted mesogens interact with their bulk counterparts via the same potential given in Eq. (1). The anchoring strength of bulk mesogens is controlled by the areal grafting density $\rho_{g}^{\prime}=N_{\mathrm{sh}} / A^{\prime}$, where $N_{\mathrm{sh}}$ is the number of mesogens in the grafted shell and $A^{\prime}$ is the surface area of a central sphere. For notational convenience and later reference we also introduce the grafting density ratio $\eta=\rho_{g} / \rho$. Grafted mesogens are distributed uniformly across the surface of the colloid as depicted schematically in Fig. 3.

\section{A. Homeotropic anchoring}

Starting with the case of homeotropic anchoring it is important to emphasize that grafted mesogens are always collinear with the local surface normal of the colloid. Therefore, there will be a instantaneous torque exerted on the decorated colloid on account of the interaction between grafted and bulk mesogens. On average, however, this torque will be zero.

Plots of the local nematic order parameter and the local director field in Fig. 17 for various grafring density ratios show a number of interesting structural changes. At the lowest grafting density ratio $\eta=0.26$ respective plots of both quantities reveal a rather diffuse region of low nematic order into which the colloid is embedded. Increasing $\eta$ to 0.41

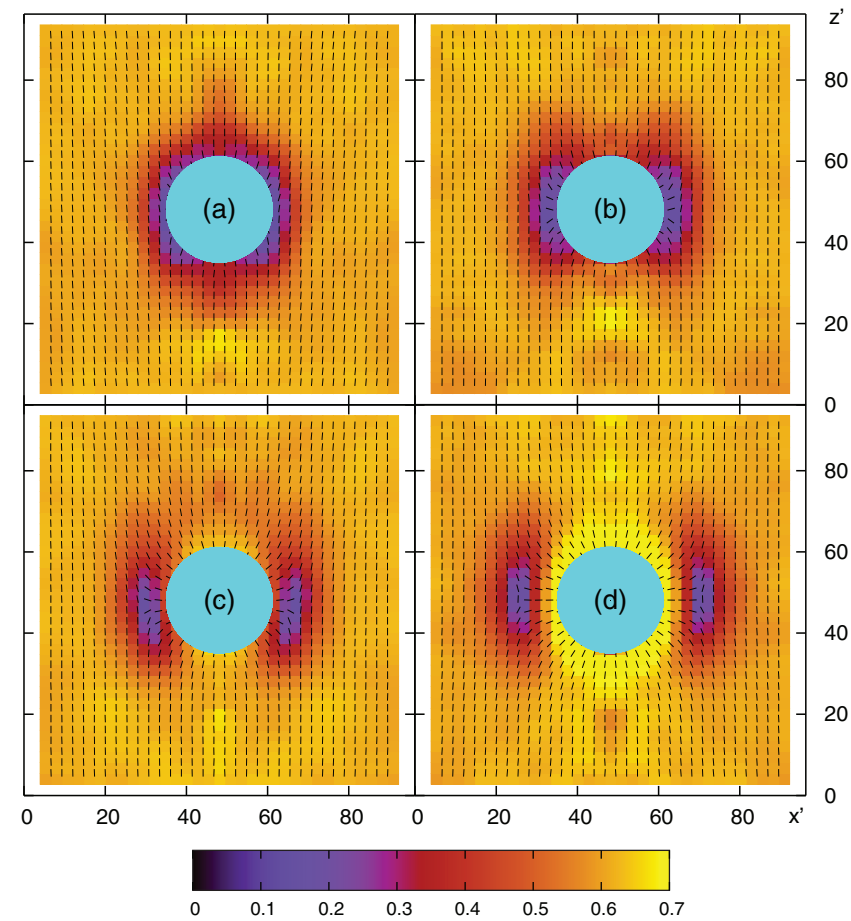

FIG. 17. As Fig. 11 but for a decorated colloid at various grafting density ratios $\eta=0.26$ (a), $\eta=0.51$ (b), $\eta=0.94$ (c), and $\eta=1.93$ (d). 


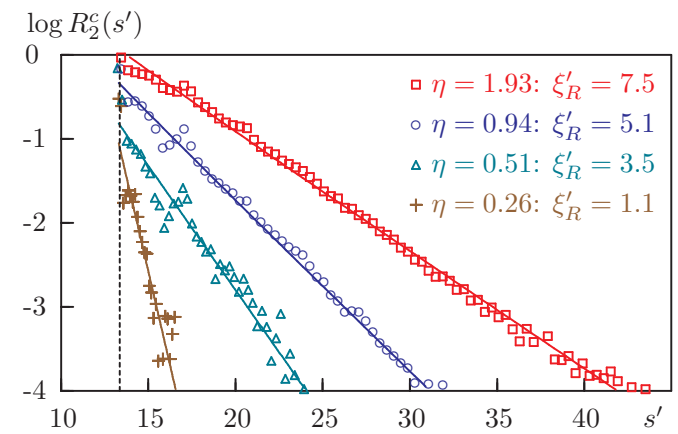

FIG. 18. Semi-logarithmic plots of the connected mesogen-colloid pair correlation function $R_{2}^{c}\left(s^{\prime}\right)$ for $s^{\prime} \geq R_{c}^{\prime}$ and various grafting density ratios $\eta$. Associated coherence lengths $\xi_{R}$ correspond to the slopes of the straight lines also shown in the figure. The dashed vertical line corresponds to $s^{\prime}=R_{c}^{\prime}$.

we notice from Fig. 17(b) that the diffuse region has become somewhat more centered on the equator of the colloid. This effect becomes more pronounced in Fig. 17(c) for $\eta=0.94$. In addition, we see from that figure that regions of low nematic order are removed somewhat from the surface of the colloid and that near the north and south pole nematic order increases. Finally, in plots in Fig. 17(d) for the highest $\eta=1.93$, indicate that regions of low nematic order have shrunk in size and are removed even further from the colloid's surface. The colloid itself now turns out to be embedded into a region of high nematic order.

These structural changes could reflect a variable anchoring strength. As we rationalised already in Sec. IV, a measure of anchoring strength is provided by the coherence length $\xi_{R}$ introduced in Eq. (13). To that end we are interested in the slope of the mesogen-colloid pair correlation function $R_{2}^{c}(s)$. Plots in Fig. 18 for different values of $\eta$ indicate that the behavior of $R_{2}^{c}(s)$ for large $s$ depends markedly on $\eta$ as reflected by a coherence length changing by more than a factor of 7 between $\eta=0.26$ and $\eta=1.93$. Notice the difference between this variation of the coherence length and the insensitivity of the corresponding $\xi_{R}$ with respect to $f_{c}^{\prime}$ for the soft colloid indicated in Fig. 14. Because $\xi_{N}$ is constant the ratio $\xi_{R} / \xi_{N}$ increases with increasing $\eta$ such that extrapolation length $\xi_{e}$ decreases. Because $\xi_{e}$ is inversely proportional to $W$, the increase of $\xi_{R}$ with $\eta$ reflects a larger strength of anchoring as we increase the grafting density.

To gain deeper insight into structural changes displayed in Fig. 17, we consider plots of the local density $\rho^{\prime}\left(s^{\prime}\right)$ in Fig. 19 for various grafting density ratios. Because of repulsive mesogen-colloid interactions and the interaction between grafted and bulk mesogens, one anticipates layering of the bulk mesogens in the vicinity of the colloid. Layering manifests itself as maxima in $\rho^{\prime}\left(s^{\prime}\right)$. In the limit of large $s^{\prime}, \rho^{\prime}\left(s^{\prime}\right)$ should approach the bulk density $\rho^{\prime}$. As one can see from Fig. 19 , for the lowest grafting density ratio, $\eta=0.51, \rho^{\prime}\left(s^{\prime}\right)$ exhibits one pronounced peak located at $s^{\prime} \approx R_{c}^{\prime}$. This can be interpreted as layering of bulk mesogens that have penetrated in between the grafted mesogens and thus are exposed to both soft repulsive and attractive interactions with grafted mesogens. As $\eta$ increases, this first peak in $\rho^{\prime}\left(s^{\prime}\right)$ diminishes, but a second peak appears at a larger value $s^{\prime}=17$. This can be

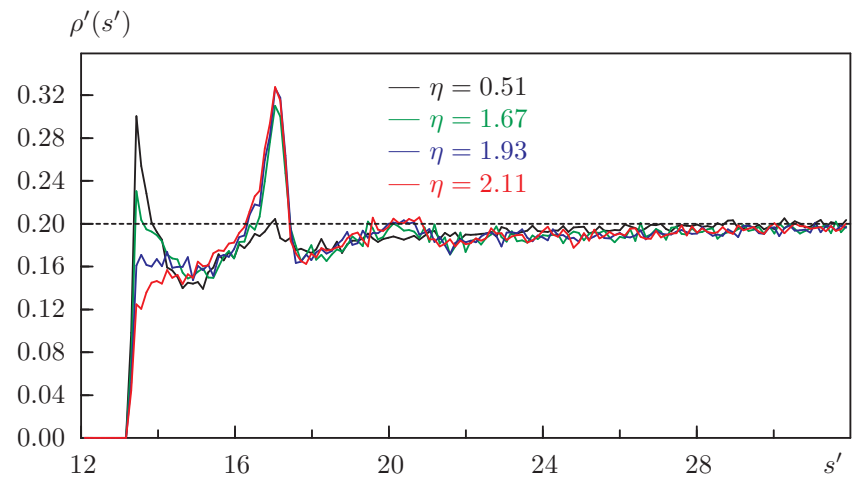

FIG. 19. Plots of the local density $\rho^{\prime}\left(s^{\prime}\right)$ as a function of a distance $s^{\prime}$ from a center of the colloid. Data are shown for various grafting density ratios $\eta$ indicated in the figure. Horizontal dashed line represents bulk density $\rho^{\prime}$.

understood because at larger grafting density ratio it becomes more difficult for bulk mesogens to penetrate into the shell of grafted mesogens. This trend continues with increasing grafting density ratio, as the plots in Fig. 19 show. It is also noteworthy that the second peak in $\rho^{\prime}\left(s^{\prime}\right)$ is rather insensitive to an increase of $\eta$ once it appears. This implies that this outer monolayer is already complete when it appears. One also sees a very weak third layer centered on $s^{\prime} \approx 20$. This layer is rather diffuse because it corresponds to bulk mesogens adsorbed on the layer of bulk mesogens at smaller $s^{\prime} \approx 17$. Both layers of bulk mesogens are rather mobile which is reflected by a quite diffuse layer at $s^{\prime} \approx 20$. The layer located at $s^{\prime} \approx 17$ is much more structured because grafted mesogens are immobilized. Regardless of the grafting density ratio $\rho^{\prime}\left(s^{\prime}\right)$ approaches the bulk density at large $s^{\prime}$ according to one's expectation.

Another structural feature that we already notice from Figs. 17(b)-17(d) is an outward shift of regions of low nematic order entangling the decorated colloid. Plots of the distance $R_{d}^{\prime}$ of the minimum of nematic order as a function of $\eta$ in Fig. 20 exhibit a monotonic increase of $R_{d}^{\prime}$ with $\eta$ very similar to what we observed earlier for the same quantity but as a function of $f_{c}^{\prime}$ controlling the strength of interaction between a mesogen and a soft colloid. Notice in particular that both curves in Figs. 12 and 20 level off for sufficiently large values of $f_{c}^{\prime}$ and $\eta$, respectively. This is particularly noteworthy because the same behavior of $R_{d}^{\prime}$ is caused by increasing the effective size of the colloid at constant anchoring strength in the case of the soft colloid, whereas both an increase of the

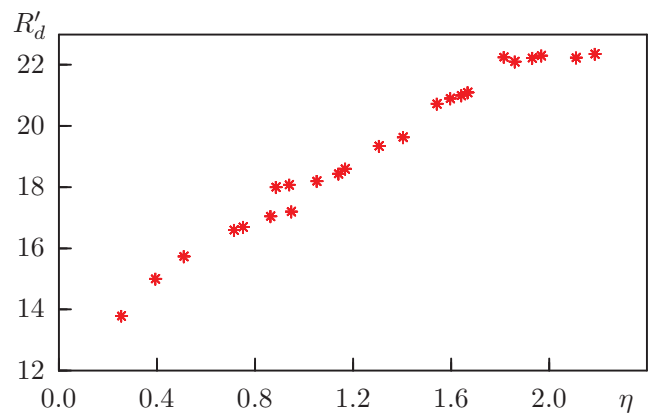

FIG. 20. As Fig. 12, but for the decorated colloid and as a function of grafting density ratio $\eta$. 
effective size of the colloid and the increase of an anchoring strength contribute to the increase of $R_{d}^{\prime}$ for a decorated colloid. Notice also that the data shown in Fig. 20 cannot be represented by a single continuous curve. In fact, the discontinuities visible in Fig. 20 occur close to those positions where the plot of the local density in Fig. 19 exhibits distinct peaks. This can be understood if one realises that the appearance of these peaks occurs rather abruptly with increasing grafting density ratio. In practice, it turned out that simulations for those values of $\eta$ where the discontinuities appear in Fig. 20 are rather unstable in the sense that the system tends to oscillate between neighboring branches of the plot in Fig. 20.

\section{B. Planar anchoring}

As already discussed in Sec. II we focus on two specific planar anchoring scenarios, namely the azimuthal and longitudinal ones, shown in Figs. 3(b) and 3(c), respectively. Both scenarios have cylindrical symmetry [contrary to the spherically symmetric homeotropic anchoring, Fig. 3(a)] with the symmetry axis marked as $\widehat{\mathbf{c}}$. Obviously, the distortion of the director field near the colloid's surface will be minimal when the easy axis $\widehat{\mathbf{a}}$ is parallel to the director $\widehat{\mathbf{n}}$. This is expected when $\widehat{\mathbf{c}} \mid \widehat{\mathbf{n}}$ for the azimuthal arrangement and $\widehat{\mathbf{c}} \perp \widehat{\mathbf{n}}$ for the longitudinal one. These particular arrangements of the decorated colloid with respect to the director are shown in Figs. 3(b) and 3(c). Therefore, when a decorated colloid is immersed into LC medium, one expects its gradual reorientation until this equilibrium state with minimal free energy is reached.

In the case of our simulation setup, the decorated colloid is fixed in space, whereas it is the director of the spontaneously assembled bulk nematic phase that reorient itself until the equilibrium state is achieved. We monitor this reorientation via the evolution of the squared components of the director $n_{x}^{2}, n_{y}^{2}$, and $n_{z}^{2}$, where $\widehat{\mathbf{n}}=n_{x} \widehat{\mathbf{i}}+n_{y} \widehat{\mathbf{j}}+n_{z} \widehat{\mathbf{k}}$. Because $\widehat{\mathbf{n}}$ is a unit vector $n_{x}^{2}+n_{y}^{2}+n_{z}^{2}=1$. The evolution of $n_{x}^{2}, n_{y}^{2}$ and $n_{z}^{2}$ alongside with the nematic order parameter $S_{2}$ are shown in Fig. 21 for the case of azimuthal anchoring at $\eta=1.0$. The $\mathrm{z}$-axis is fixed parallel to the symmetry axis $\widehat{\mathbf{c}}$ [as shown in Fig. 3(b)]. As one can see in Fig. 21, the nematic order already saturates at $t^{\prime} \approx 5000$, but the director of the bulk phase is in the $\mathrm{x}-\mathrm{z}$ plane at that time. The reorientation of the director towards the $\mathrm{z}$-axis is much slower and it is finally complete at $t^{\prime} \approx 50000$. In the equilibrium configuration the nematic director $\widehat{\mathbf{n}}$ is parallel to the symmetry axis $\widehat{\mathbf{c}}$ of the colloid.

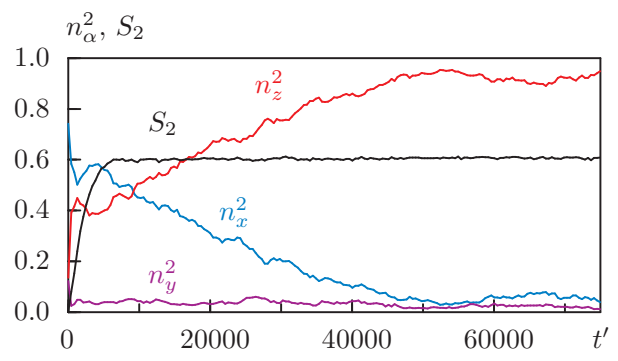

FIG. 21. Plot of squared components of the global nematic director $n_{a}^{2}$ $(a=x, y$, or $z)$ and of the nematic order parameter $S_{2}$ as a function of time $t^{\prime}$ for a grafting density ratio $\eta=1$.

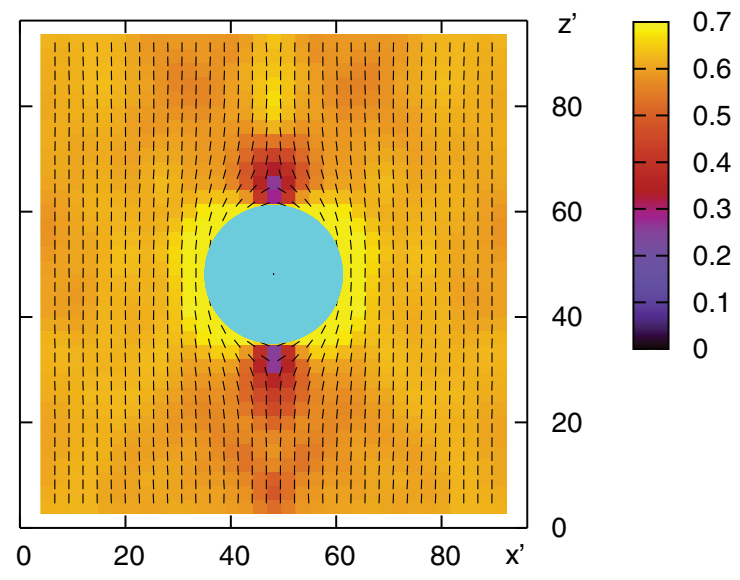

FIG. 22. As Fig. 11, but for a decorated colloid with azimuthal planar anchoring and grafting density ratio $\eta=2$. The circular turquoise region at the center represents the sphere of radius $R_{c}^{\prime}$, see Eq. (14).

For the case of azimuthal anchoring, plots of the local nematic order parameter and the director field are presented in Fig. 22. As one can see, nematic order is smallest in small regions on the colloids north and south pole, therefore reflecting the well-known boojum defect topology. As we have checked, quite similar plots are obtained for different grafting density ratios in the range $0.5 \leq \eta \leq 2$. More details on the fine structure of this defect will be given below.

Similar to the decorated colloid with locally homeotropic anchoring we studied the mesogen-colloid pair correlation function for different grafting density ratios in Fig. 23. Because of its definition in Eq. (11) $R_{2}(s)$ can become negative if $\widehat{\mathbf{e}}_{i}$ and $\widehat{\mathbf{s}}_{i}$ are orthogonal. Therefore, we consider the magnitude of its connected counterpart $R_{2}^{s}(s)$ in the semilogarithmic plot in Fig. 23. Unlike its counterparts presented in Fig. 18, the decay of $\left|R_{2}^{c}(r)\right|$ does not seem to depend on the grafting density ratio, but is characterized by an almost constant coherence length $\xi_{R}^{\prime} \approx 5.9$. This coherence length is, however, of the same order of magnitude as that obtained for homeotropic anchoring. The insensitivity of the coherence length in the planar anchoring case is presumably caused by the flatness of the colloid's surface in this case. As a result, soft steric interactions between grafted and bulk mesogens are

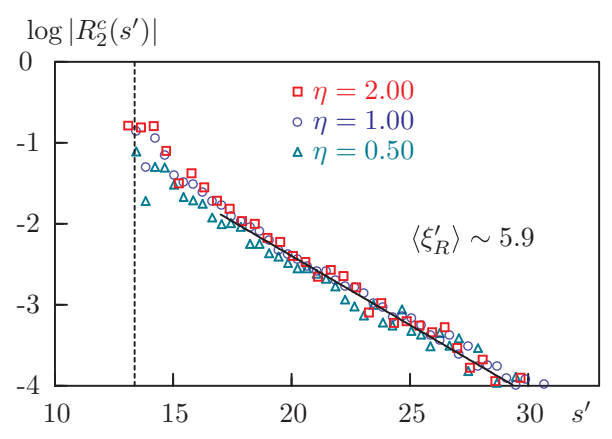

FIG. 23. Semi-logarithmic plot of the magnitude of the connected mesogencolloid pair correlation function $R_{2}^{c}\left(s^{\prime}\right)$ as a function of distance $s^{\prime}$ from the colloid's center and for different grafting density ratios indicated in the figure. From the large-distance slope of the curves the radial coherence length (also given in the figure) is determined. 
(a)

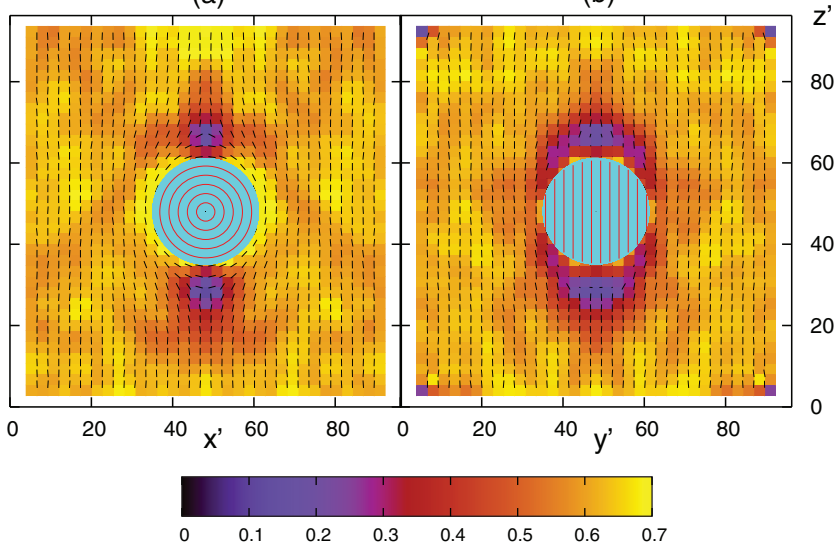

FIG. 24. As Fig. 11, but for the biaxial boojum defect. (a) Projection onto the $\mathrm{x}-\mathrm{z}$ plane, (b) projection onto the $\mathrm{y}-\mathrm{z}$ plane. The turquoise region at the center represents the colloid. Lines in the interior of the colloid reflect the direction of the $\widehat{\mathbf{n}}$ in these different projections which is orthogonal to the paper plane (a) or lies in the paper plane (b).

supressed and anchoring is maintained solely due to attractive force [see, Eq. (1)]. Therefore, the grafted mesogens in the case of planar anchoring serve only to enforce the surface of the decorated colloid, whereas in the homeotropic case bulk mesogens could move in between the grafted ones, depending on the grafting density ratio.

Let us consider now the case of longitudinal planar anchoring. As is evident from the plot in Fig. 3(b), there will be two symmetry axes when such a decorated colloid is immersed in a bulk nematic phase. One of these coincides with the global director $\widehat{\mathbf{n}}$, whereas the other one is defined by the symmetry axis $\widehat{\mathbf{c}}$ of the colloid. It is expected to be perpendicular to $\widehat{\mathbf{n}}$ in the equilibrium state. This is indeed the case and therefore it is instructive to consider two different projections of the local nematic director and the local order parameter. Taking the global director $\widehat{\mathbf{n}}$ to coincide with the $\mathrm{z}$-axis and the symmetry axis of the decorated colloid $\widehat{\mathbf{c}}$ to coincide with the y-axis, projections of the local nematic order parameter onto the $\mathrm{x}$ - $\mathrm{z}$ plane (y-axis is orthogonal to the paper plane) shown in Fig. 24(a), reveal a defect topology very much reminiscent of the boojum defect topology shown in Fig. 22. This picture changes if one projects the local nematic order parameter onto the y-z plane, as one can see from Fig. 24(b). In this case one can still see features of a boojum topology. However, regions of lowest nematic order are embedded into larger, "handle"-like, regions of low but slightly larger nematic order located at "caps" sitting on the north and south pole of the colloid, respectively. Notice also that for the regions of lowest nematic order the local director field is perpendicular to the paper plane, following the easy axis $\widehat{\mathbf{a}}$ on the colloid's surface.

To compare boojum defects obtained for azimuthal and longitudinal planar anchoring we present plots of the local order parameter and the local director with enhanced resolution in Fig. 25. Notice also that plots in Fig. 25 are projections onto the $\mathrm{x}-\mathrm{y}$ plane. To make these projections we consider mesogens located in a thin slice of a certain thickness $\Delta z^{\prime} \approx 2-4$ located at various distances from the colloid's surface. For (a)

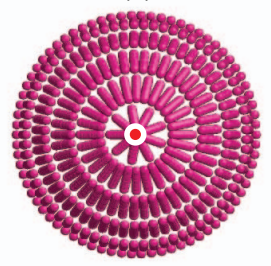

(b)

(c)

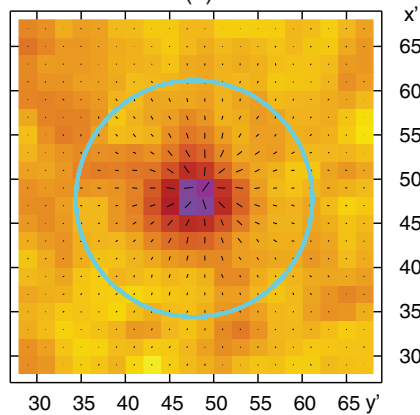

$\begin{array}{llllllll}30 & 35 & 40 & 45 & 50 & 55 & 60 & 65 y^{\prime}\end{array}$

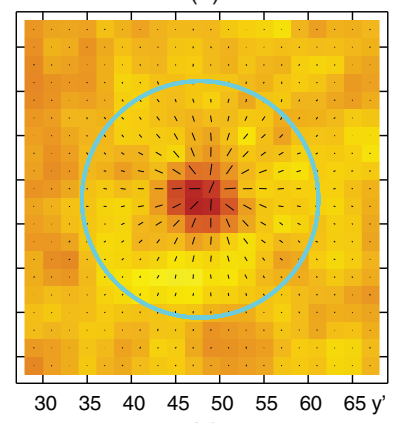

(d)

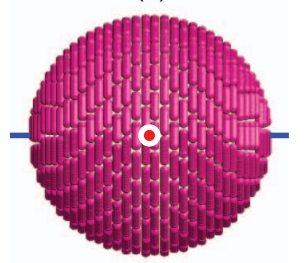

(e)

(f)

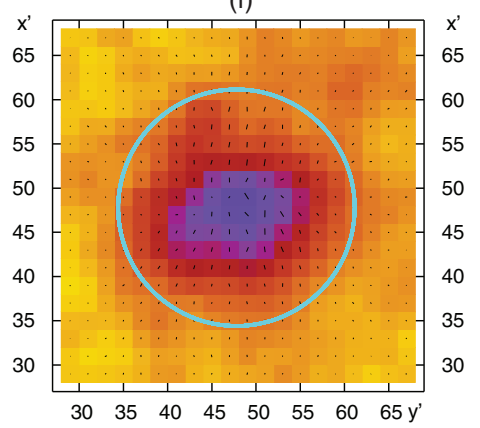

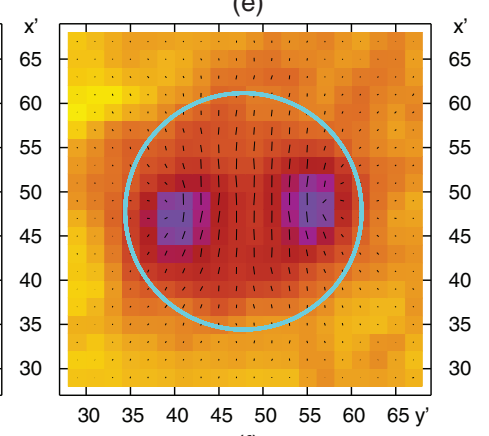

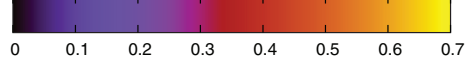

FIG. 25. As Fig. 11, but for boojum defects forming for azimuthal [(a)-(c)] and longitudinal [(d)-(f)] planar anchoring. Plots are projections on the $\mathrm{x}$ y plane perpendicular to the global nematic director (see text). Images (a) and (d) are snapshots of grafted mesogens in each case and illustrate the arrangement of the easy axis on the colloid's surface. Plots in (b) and (e) have been obtained for mesogens located in slices close to the colloid surface whereas plots in (c) an (f) correspond to slices at a larger distance from the colloid's surface.

azimuthal planar anchoring the projected local director field appears to have radial symmetry [see Fig. 25(a)]. The plot in Fig. 25(a) illustrates a quasi 2D hedgehog defect forming at the center and similar to the one displayed in Fig. 11(c). Considering next mesogens in a slice removed further from the colloid's surface [see Fig. 25(c)], the circular region with radial pattern for local director shrinks. If the slice is located at an even larger distance from the colloid's surface, the quasi 2D hedgehog defect disappears altogether.

This situation is different for the case of longitudinal planar anchoring [Figs. 25(d)-25(f)]. In this case the arrangement of the easy axis is locally planar [image (d)]. The local director near the surface coincides with the easy axis $\widehat{\mathbf{a}}$. However, the mismatch between the near-surface director and the global director $\widehat{\mathbf{n}}$ causes a defect to form manifested as regions of low nematic order in Fig. 25(e). Because the plot in Fig. 25(e) is a projection onto the $x-y$ plane these defect regions have the shape of handles in a three-dimensional representation. The slice cut at a larger distance from the colloid's 
surface [see Fig. 25(f)] cuts through the top of the handle. In this case the two regions of low order merge into a single extended region. This shape of the defect can now also be seen in Fig. 24. It has been referred to earlier ${ }^{26}$ as split-core boojum. The combined analysis presented in Figs. 24 and 25 illustrates the biaxiality of the split-core boojum defect topology.

\section{CONCLUSIONS}

The present study is the first one in which a coarsegrained model is used to study defect topologies that emerge if a spherical colloidal particle is immersed in the nematic LC host phase. Our study is based upon molecular dynamics simulations in the canonical ensemble where we maintain a fixed temperature by rescaling velocities during the course of the simulation. On account of the coarse-grained nature of our models, we have to determine length and energy scales of the host fluid first. Assuming the host fluid to correspond to PAA, we establish the energy scale by computing ratios of the elastic constants corresponding to the usual splay, twist, and bend deformation of the director field and relating them to the experimentally known values for PAA. To establish the length scale characteristic of our model we consider the mesogen-mesogen pair orientation correlation function and determine the coherence length from its largedistance decay. This coherence length turns out to correspond to the assembly of 7-10 individual molecules, which seems to be a reasonable number in view of our coarse-grained approach.

As our main focus is on the impact of a colloidal particle immersed in a nematic host phase, two different model colloids are considered in this work, namely, a so-called soft colloid and a decorated one. The soft colloid, which we take to represent, for example, a main-chain liquid crystal dendrimer, consists of a spherical region of variable thickness in which bulk mesogens are exposed to an orienting field of variable strength. Hence, during the course of the simulation, these bulk mesogens are essentially free to enter or leave the interior of the soft colloid. Thus, our model is similar in spirit to the one used by Tanaka and $\mathrm{Araki}^{41}$ in which the fluid in a certain region of space is assumed to have a different viscosity compared to the remainder of the system. However, in their case only the translational viscosity is affected.

In a similar way, we set up the soft colloid as a region in space in which an orienting field acts upon the mesogens. This will affect not only the translational viscosity as in Tanaka and Araki's case but also the rotational viscosity. If this orienting field has radial symmetry we observe the well-known Saturn ring defect topology ${ }^{18,23,25,61}$ outside the soft colloid. In addition, we also reproduce internal ring defects inside the soft colloid also reported earlier. ${ }^{43,62,63}$ To the best of our knowledge, this work is the first study in which inner and outer defect rings exist simultaneously.

By tuning the strength of the external field or the thickness of the shell upon which it acts, we show that the radius of the inner and outer defect rings can be shifted with respect to the respective surface of the soft colloid. At the same time, the coherence length of the mesogen-colloid pair correlation function remains unchanged. Both results are consistent if one concludes that the anchoring strength remains constant but the effective size of the soft colloid changes if the strength of the external field and the shell thickness are varied.

If the external field induces tangential anchoring, the defect topology can be understood as a combination of two separate ones. On the outside of the colloid, we observe the well-known boojum defect topology $y^{21,25,26}$ consisting of regions of low nematic order centered on the colloid's north and south pole, respectively. In addition, an extended region of low nematic order inside the colloid exists, connecting regions of low nematic order on its outside. Notice also that everywhere inside the colloid the locally tangential orientation of the mesogens is preserved.

The other model that we consider is that of a colloid with a repulsive core, which is decorated with immobilised mesogens such that they are attached to the outer surface of the colloid. As before for the soft colloid locally homeotropic and planar alignment of the grafted mesogens are considered. In both cases the interaction strength between the decorated colloid and a bulk mesogen is controlled by the grafting density of mesogens on the colloid surface.

In the case of homeotropic anchoring of the grafted mesogens we find that at low grafting density bulk mesogens can penetrate in between the shell of grafted mesogens. The higher the grafting density the less capable are bulk mesogens to move into this shell. We find that the coherence length of the mesogen-colloid pair correlation function varies significantly with grafting density thus indicating that at higher grafting density the anchoring strength is higher than at lower grafting density. At higher grafting density bulk mesogens tend to form layers adsorbed onto the grafted mesogens. However, whereas layering is clearly visible for the first layer, the second one is already quite diffuse. This layering phenomenon is seen as a direct consequence of the increasing anchoring strength with increasing grafting density.

If the colloid is instead decorated with mesogens that are grafted with a locally planar orientation with respect to its surface, the anchoring strength again turns out to be unaffected by the grafting density as we conclude from the large-distance slope of the mesogen-colloid pair correlation function. However, here we observe another interesting defect topology depending on details of the planar anchoring with respect to both the colloid's inherent symmetry axis and the direction of the global nematic director. It consists of a boojum-like defect topology with biaxial symmetry. This biaxiality of a boojum defect topology is reported here for the first time but should, in principle, be accessible to other theoretical or even experimental techniques. A necessary prerequisite for a possible determination of such a biaxial boojum defect in experiments would be precise control of the easy axes on the colloid's surface.

The present study does not address the issue of computational efficiency of mesoscopic models as opposed to their semi-atomistic counterparts (e.g., the Gay-Berne model). In view of the fact that both types of models involve a lot of parameters and that the phase diagram will be different for each combination of these parameters a meaningful comparison of the two types of models seems computationally very demanding if not at all prohibitive. 


\section{ACKNOWLEDGMENTS}

The authors acknowledge financial support by EU under IRSES Project STCSCMBS 268498 and are grateful to S. Žumer and M. Tasinkevych for fruitful discussions.

${ }^{1}$ M. Kleman and O. D. Lavrentovich, Soft Matter Physics: An Introduction (Springer, 2003), p. 637.

${ }^{2}$ P. Poulin, H. Stark, T. C. Lubensky, and D. A. Weitz, Science 275, 1770 (1999).

${ }^{3}$ S. Kurihara, K. Ohta, T. Oda, R. Izumi, Y. Kuwahara, T. Ogata, and S.-N. Kim, Sci. Rep. 3, 2167 (2013)

${ }^{4}$ B. Senyuk, Q. Liu, S. He, R. D. Kamien, R. B. Kusner, T. C. Lubensky, and I. I. Smalyukh, Nature 493, 200 (2013).

${ }^{5}$ J. Planer, Condens. Matter Phys. 13, 37001 (2010).

${ }^{6}$ I. Smalyukh, A. Kuzmin, A. Kachynski, P. Prasad, and O. Lavrentovich, Appl. Phys. Lett. 86, 021913 (2005)

${ }^{7}$ I. Musevic, M. Skarabot, U. Tkalec, M. Ravnik, and S. Zumer, Science 313, 954 (2005).

${ }^{8}$ K. Takahashi, M. Ichikawa, and Y. Kimura, Phys. Rev. E 77, 020703 (2005).

${ }^{9}$ M. Skarabot and I. Musevich, Soft Matter 6, 5476 (2010).

${ }^{10}$ H. Stark, Phys. Rep. 351, 387 (2001).

${ }^{11}$ Defects in Liquid Crystals: Computer Simulations, Theory and Experiments, NATO Science Series, edited by O. D. Lavrentovich, P. Pasini, C. Zannoni, and S. Žumer (Kluwer, 2001), Vol. 43.

${ }^{12}$ M. Tasinkevych and D. Andrienko, Condens. Matter Phys. 13, 33603 (2010).

${ }^{13}$ V. M. Pergamenshchik and V. A. Uzunova, Phys. Rev. E 83, 021701 (2011).

${ }^{14}$ P. Poulin, V. Cabuil, and D. A. Weitz, Phys. Rev. Lett. 79, 4862 (1997).

${ }^{15}$ F. C. Frank, Discuss. Faraday Soc. 25, 19 (1958).

${ }^{16}$ T. C. Lubensky, D. Pettey, N. Currier, and H. Stark, Phys. Rev. E 57, 610 (1998).

${ }^{17}$ J. I. Fukuda, M. Yoneya, and H. Yokoyama, Phys. Rev. E 65, 041709 (2002).

${ }^{18}$ O. V. Kuksenok, R. W. Ruhwandl, S. V. Shiyanovskii, and E. M. Terentjev, Phys. Rev. E 54, 5198 (1996).

${ }^{19}$ R. W. Ruhwandl and E. M. Terentjev, Phys. Rev. E 56, 5561 (1997).

${ }^{20}$ S. Grollau, E. B. Kim, O. Guzman, N. L. Abbott, and J. J. de Pablo, J. Chem. Phys. 119, 2444 (2003)

${ }^{21}$ M. Melle, S. Schlotthauer, M. G. Mazza, S. H. L. Klapp, and M. Schoen, J. Chem. Phys. 136, 194703 (2012).

${ }^{22}$ J. L. Billeter and R. A. Pelcovits, Phys. Rev. E 62, 711 (2000)

${ }^{23}$ D. Andrienko, G. Germano, and M. P. Allen, Phys. Rev. E 63, 041701 (2001)

${ }^{24}$ J. A. Moreno-Razo, E. J. Sambriski, G. M. Koenig, E. Diaz-Herrera, N. L. Abbott, and J. J. de Pablo, Soft Matter 7, 6828 (2011).

${ }^{25}$ G. E. Volovik and O. D. Lavrentovich, Sov. Phys. JETP 58, 1159 (1983); available at http://www.jetp.ac.ru/cgi-bin/e/index/e/58/6/p1159?a=list.

${ }^{26}$ M. Tasinkevych, N. M. Silvestre, and M. M. Telo da Gama, New J. Phys. 14, 073030 (2012)

${ }^{27}$ J. G. Gay and B. J. Berne, J. Chem. Phys. 74, 3316 (1981).

${ }^{28}$ S. Hess, and B. Su, Z. Naturforsch. A 54, 559 (1999); available at http://www.znaturforsch.com/aa/v54a/c54a.htm.
${ }^{29}$ J. S. Lintuvuori and M. R. Wilson, J. Chem. Phys. 128, 044906 (2008).

${ }^{30}$ J. M. Ilnytskyi, J. S. Lintuvuori, and M. R. Wilson, Condens. Matter Phys. 13, 33001 (2010).

${ }^{31}$ R. Berardi, J. S. Lintuvuori, M. R. Wilson, and C. Zannoni, J. Chem. Phys. 135, 134119 (2011).

${ }^{32}$ S. D. Peroukidis, A. G. Vanakaras, and D. J. Photinos, Phys. Rev. E 84, 010702 (2011)

${ }^{33}$ J. S. Lintuvuori and M. R. Wilson, Phys. Chem. Chem. Phys. 11, 2116 (2009).

${ }^{34}$ J. M. Ilnytskyi, Condens. Matter Phys. 16, 43004 (2013).

${ }^{35}$ M. Draper, I. M. Saez, S. J. Cowling, P. Gai, B. Heinrich, B. Donnio, D. Guillon, and J. W. Goodby, Adv. Funct. Mater. 21, 1260 (2011)

${ }^{36}$ H. K. Bisoyi and S. Kumar, Chem. Soc. Rev. 40, 306 (2011).

${ }^{37}$ I. M. Saez and J. W. Goodby, J. Mater. Chem. 15, 26 (2005).

${ }^{38}$ B. Donnio, S. Buathong, I. Bury, and D. Guillon, Chem. Soc. Rev. 36, 1495 (2007).

${ }^{39}$ I. I. Smalyukh, Y. Lansak, N. A. Clark, and R. P. Trivedi, Nat. Mater. 9, 139 (2010).

${ }^{40}$ J. S. Evans, Y. Sun, B. Senyuk, P. Keller, V. M. Pergamenshchik, T. Lee, and I. I. Smalyukh, Phys. Rev. Lett. 110, 187802 (2013).

${ }^{41}$ H. Tanaka and T. Araki, Phys. Rev. Lett. 85, 1338 (2000).

${ }^{42}$ T. Araki and H. Tanaka, J. Phys.: Condens. Matter 18, L193 (2006).

${ }^{43}$ C. Chiccoli, P. Pasini, I. Feruli, and C. Zannoni, in Defects in Liquid Crystals: Computer Simulations. Theory and Experiments, Nato Science Series II, edited by D. Lavrentovich, P. Pasini, C. Zannoni, and S. Žumer (Kluwer, 2002), Vol. 80, Chap. XV, pp. 87-112, 344.

${ }^{44}$ D. Andrienko and M. P. Allen, Phys. Rev. E 61, 504 (2000).

${ }^{45}$ M. Kleman and O. D. Lavrentovich, Philos. Mag. 86, 4117 (2006).

${ }^{46}$ T. Kihara, J. Phys. Soc. Jpn. 6, 289 (1951).

${ }^{47}$ P. J. Hoogerbrugge and J. M. V. A. Koelman, Europhys. Lett. 19, 155 (1992).

${ }^{48}$ P. Español and P. Warren, Europhys. Lett. 30, 191 (1995).

${ }^{49}$ R. D. Groot and P. B. Warren, J. Chem. Phys. 107, 4423 (1997).

${ }^{50}$ M. R. Wilson, J. M. Ilnytskyi, L. M. Stimson, and Z. E. Hughes, in Computer Simulations of Liquid Crystals and Polymers, edited by P. Pasini, C. Zannoni, and S. Zumer (Kluwer, 2004), pp. 57-78.

${ }^{51}$ Z. E. Hughes, M. R. Wilson, and L. M. Stimson, Soft Matter 1, 436 (2005).

${ }^{52}$ Z. E. Hughes, L. M. Stimson, H. Slim, J. S. Lintuvuori, J. M. Ilnytskyi, and M. R. Wilson, Comput. Phys. Commun. 178, 724 (2008).

${ }^{53} \mathrm{H}$. Steuer, S. Hess, and M. Schoen, Physica A 328, 322 (2003).

${ }^{54}$ M. P. Allen, M. A. Warren, M. R. Wilson, A. Sauron, and W. Smith, J. Chem. Phys. 105, 2850 (1996).

${ }^{55}$ N. H. Phuong, G. Germano, and F. Schmid, J. Chem. Phys. 115, 7227 (2001).

${ }^{56}$ W. H. de Jeu and W. A. P. Classen, J. Chem. Phys. 67, 3705 (1977); 68, 102 (1978); W. H. de Jeu, W. A. P. Classen, and A. M. J. Sprujit, Mol. Cryst. Liq. Cryst. 37, 269 (1976).

${ }^{57}$ A. Srivastava and S. Singh, J. Phys.: Condens. Matter 16, 7169 (2004).

${ }^{58}$ T. J. H. Vlugt, R. Krishna, and B. Smit, J. Phys. Chem. B 103, 1102 (1999).

${ }^{59} \mathrm{C}$. Zannoni, The Molecular Physics of Liquid Crystals (Academic, London, 1979), Chap. 3, p. 76.

${ }^{60}$ B. Chu, C. S. Bak, and F. L. Lin, Phys. Rev. Lett. 28, 1111 (1972).

${ }^{61}$ Y. Gu and N. L. Abbott, Phys. Rev. Lett. 85, 4719 (2000).

${ }^{62}$ O. D. Lavrentovich and E. M. Terentjev, Sov. Phys. JETP 64, 1237 (1986).

${ }^{63}$ A. V. Kovalchuk, M. V. Kurik, O. D. Lavrentovich, and V. V. Sergan, Sov. Phys. JETP 67, 1065 (1988) 